SISSA 38/97/EP

NSF-ITP-97-034

August 18, 2021

\title{
$N$-species Stochastic models with boundaries and quadratic algebras
}

\author{
F. C. ALCARAZ円 \\ Departamento de Física \\ Universidade Federral de São Carlos \\ 13565-905 São Carlos, SP, Brazil \\ S. DASMAHAPATRA \\ Department of Mathematics \\ City University \\ London EC1 V OHB, UK \\ V. RITTENBERG \\ SISSA, Via Beirut 2-4 \\ Trieste I-34014 Italy \\ and \\ Physikalisches Institut \\ Universität Bonn \\ D-53115 Bonn, Germany
}

\footnotetext{
${ }^{1}$ Partially supported by CNPQ and FAPESP-Brazil

${ }^{2}$ Partially supported by the EPSRC grant GRJ 25758

${ }^{3}$ Work done under partial support of the EC TMR Programme grant FMXR-CT960012
} 


\begin{abstract}
Stationary probability distributions for stochastic processes on linear chains with closed or open ends are obtained using the matrix product Ansatz. The matrices are representations of some quadratic algebras. The algebras and the types of representations considered depend on the boundary conditions. In the language of quantum chains we obtain the ground state of $N$-state quantum chains with free boundary conditions or with non-diagonal boundary terms at one or both ends. In contrast to problems involving the Bethe Ansatz, we do not have a general framework for arbitrary $N$ which when specialized, gives the known results for $N=2$; in fact, the $N=2$ and $N>2$ cases appear to be very different.
\end{abstract}




\section{Introduction}

The aim of this paper is to present in a systematic way the application of quadratic algebras to obtain the steady state probability distribution of onedimensional stochastic processes with boundaries. In this section we first present the problem and describe what is already known about the subject giving the relevant references and then proceed by giving the contents of the next sections. The list of possible physical applications of our results include interface growth [1], boundary induced phase transitions [2, 3, 4, 5], the dynamics of shocks [6] or traffic flow [7].

Consider a linear chain with $L$ sites. On each site $k$ we put a discrete stochastic variable $\beta_{k}$ taking values from $\{0,1, \ldots, N-1\}$. For each link $k$ between sites $k$ and $k+1$ we give transition (intensity) rates $\Gamma_{\beta_{k} \beta_{k+1}}^{\gamma_{k} \gamma_{k+1}}$ giving the probability per unit time for the transition

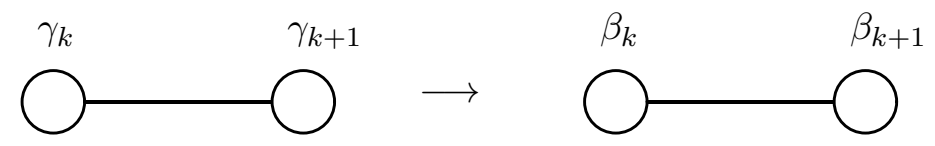

These are the bulk rates. At the left end of the chain (site 1) we also consider transition rates

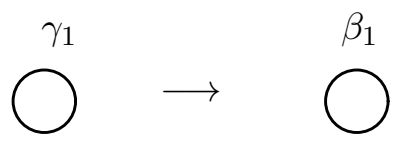

given by the $N \times N$ matrix $L_{\beta_{1}}^{\gamma_{1}}$. Similarly, at the right end of the chain (site $L)$ we take processes

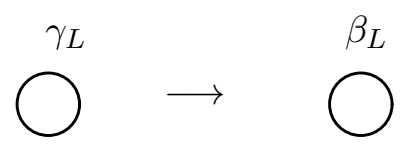

with rates $R_{\beta_{L}}^{\gamma_{L}}$. The matrices $L$ and $R$ give the boundary rates. We consider Markov processes in continuous time, which implies that the rate matrices are intensity matrices, which have the property [8]

$$
\Gamma_{\beta_{k} \beta_{k+1}}^{\beta_{k} \beta_{k+1}}=-\sum_{\substack{\gamma_{k}, \gamma_{k+1}=0 \\\left(\gamma_{k}, \gamma_{k+1}\right) \neq\left(\beta_{k}, \beta_{k+1}\right)}}^{N-1} \Gamma_{\gamma_{k} \gamma_{k+1}}^{\beta_{k} \beta_{k+1}} ; L_{\beta}^{\beta}=-\sum_{\substack{\gamma=0 \\ \gamma \neq \beta}}^{N-1} L_{\gamma}^{\beta} ; R_{\beta}^{\beta}=-\sum_{\substack{\gamma=0 \\ \gamma \neq \beta}}^{N-1} R_{\gamma}^{\beta} .
$$


Sometimes it will be convenient to write the intensity matrices (for example $R$ ) in an alternative form:

$$
R_{\beta}^{\gamma}=R_{\beta \gamma}
$$

indicating by the column $(\gamma)$ the initial state and by the row $(\beta)$ the final state. Equations (1.1) just state that the sum of the matrix elements on each column of an intensity matrix is zero. The time evolution of the probability distribution $P\left(\beta_{1}, \beta_{2}, \ldots, \beta_{L} ; t\right)=: P(\{\beta\} ; t)$ is given by the master equation

$$
\begin{aligned}
& \frac{\partial}{\partial t} P(\{\beta\} ; t)=\sum_{k=1}^{L-1} \sum_{\gamma_{k}, \gamma_{k+1}=0}^{N-1} \Gamma_{\beta_{k} \beta_{k+1}}^{\gamma_{k} \gamma_{k+1}} P\left(\beta_{1}, \beta_{2}, \ldots, \gamma_{k}, \gamma_{k+1}, \ldots, \beta_{L} ; t\right) \\
& \quad+\sum_{\gamma_{1}=0}^{N-1} L_{\beta_{1}}^{\gamma_{1}} P\left(\gamma_{1}, \beta_{2}, \ldots, \beta_{L} ; t\right)+\sum_{\gamma_{L}=0}^{N-1} R_{\beta_{L}}^{\gamma_{L}} P\left(\beta_{1}, \ldots, \beta_{L-1}, \gamma_{L} ; t\right) .
\end{aligned}
$$

Solving equation (1.3) is equivalent to finding the wave-function of an imaginary time Schrödinger equation (see [9] and references therein for the notations) which is obtained as follows. We consider an orthonormal system of states

$$
\left.|\beta\rangle\rangle=\left|\beta_{1}, \ldots \beta_{L}\right\rangle\right\rangle \quad ; \quad\langle\langle\gamma|\cdot| \beta\rangle\rangle=\prod_{j=1}^{L} \delta_{\gamma_{j} \beta_{j}},
$$

and a basis in the space of $N \times N$ matrices $E^{\alpha \beta}$ :

$$
\left(E^{\alpha \beta}\right)_{\gamma, \delta}=\delta_{\alpha, \gamma} \delta_{\beta, \delta}
$$

By $E_{k}^{\alpha \beta}$ we denote the matrix $E^{\alpha \beta}$ acting on the $k^{\text {th }}$ site. The probability distribution $P(\{\beta\} ; t)$ is mapped into a ket state:

$$
\left.|P\rangle\rangle=\sum_{\{\beta\}} P(\{\beta\} ; t)|\beta\rangle\right\rangle=\left(\begin{array}{c}
P(0,0, . ., 0 ; t) \\
P(0,0, . ., 1 ; t) \\
\vdots \\
P(N-1, N-1, . ., N-1 ; t)
\end{array}\right)
$$

and the master equation (1.3) implies the Schrödinger equation

$$
\left.\left.\frac{\partial}{\partial t}|P\rangle\right\rangle=-H|P\rangle\right\rangle
$$

where

$$
H=\sum_{k=1}^{L-1} H_{k, k+1}+H_{1}+H_{L}
$$




$$
\begin{gathered}
H_{k, k+1}=-\Gamma_{\gamma \delta}^{\alpha \beta} E_{k}^{\gamma \alpha} E_{k+1}^{\delta \beta}, \\
H_{1}=-L_{\beta}^{\alpha} E_{1}^{\beta \alpha} \text { and } H_{L}=-R_{\beta}^{\alpha} E_{L}^{\beta \alpha} .
\end{gathered}
$$

Above, and subsequently, the summation is invoked on any pair of repeated indices (raised, lowered, or mixed).

In this paper we are interested in finding the stationary probability distribution $P_{s}(\{\beta\})$ of the master equation (1.3), i.e. the ground state wave function $\left.\left|P_{s}\right\rangle\right\rangle$ of the Hamiltonian (1.8):

$$
\left.H\left|P_{s}\right\rangle\right\rangle=0 \text {. }
$$

Since $H$ is an intensity matrix with positive rates the ground-state energy is zero. Unless stated otherwise we consider unnormalized probability distributions. The interest in knowing the stationary distributions of stochastic processes with boundaries is illustrated by the extensive list of references on the subject which can be found for example in [5]. If the bulk rates give a Hamiltonian with a certain algebra or superalgebra as symmetry (see ref. [10] for many examples) the boundary terms might break this symmetry.

Recently Krebs and Sandow [11] have proven the following remarkable theorem. With $\Gamma, L, R$ matrices as above, take $N$ matrices $D_{\alpha}(\alpha=$ $0,1, \ldots, N-1)$ and $N$ matrices $X_{\alpha}(\alpha=0,1, \ldots, N-1)$ acting in an auxiliary vector space which satisfy the quadratic algebra relations 1

$$
\Gamma_{\gamma \delta}^{\alpha \beta} D_{\alpha} D_{\beta}=D_{\gamma} X_{\delta}-X_{\gamma} D_{\delta}, \quad(\gamma, \delta=0,1, \ldots, N-1) .
$$

Consider now a ket state $|0\rangle$ and a bra state $\langle 0|$ in the auxiliary space (we use the same notation suggesting the "vacuum" of quantum mechanics for reasons which will be apparent later). If these states are chosen such that the following conditions are fulfilled:

$$
\langle 0|\left(X_{\alpha}-L_{\alpha}^{\beta} D_{\beta}\right)=0 \quad \text { and } \quad\left(X_{\alpha}+R_{\alpha}^{\beta} D_{\beta}\right)|0\rangle=0,
$$

then

$$
\begin{aligned}
P_{s}(\{\beta\}) & =\left\langle 0\left|\prod_{k=1}^{L} D_{\beta_{k}}\right| 0\right\rangle \\
& =\left\langle 0\left|\prod_{k=1}^{L}\left(\sum_{\mu_{k}=0}^{N-1} D_{\mu_{k}} \delta_{\beta_{k}, \mu_{k}}\right)\right| 0\right\rangle
\end{aligned}
$$

\footnotetext{
${ }^{4}$ Observe that the quadratic algebra (1.12) is unusual since the $X_{\alpha}$ 's appear linearly. (See refs. [12] and [13] for a discussion of quadratic algebras in the mathematical literature.)
} 
is a stationary solution of the master equation (1.3). Alternatively, let us denote by $u_{\mu_{k}}(\mu=0,1, \ldots, N-1 ; k=1, \ldots, L)$ basis vectors in the vector space associated with the $k^{t h}$ site $\left(\left(u_{\mu_{k}}\right)_{\nu}=\delta_{\mu, \nu}\right.$ for any $\left.k\right)$ on which the matrices $E_{k}^{\beta \alpha}$ act (see eqs. (1.9-10)). The ground state wave-function of the Hamiltonian (1.8) has the expression

$$
\left.\left|P_{s}\right\rangle\right\rangle=\left\langle 0\left|\prod_{k=1}^{L}\left(\sum_{\mu_{k}=0}^{N-1} D_{\mu_{k}} u_{\mu_{k}}\right)\right| 0\right\rangle .
$$

Actually, the Krebs-Sandow theorem is even stronger; the matrices $\Gamma_{\gamma \delta}^{\alpha \beta}, R_{\beta}^{\alpha}$ and $L_{\beta}^{\alpha}$ in (1.8) do not have to be intensity matrices. If the Hamiltonian has an eigenvalue zero, then the wave function is given by (1.15). Moreover, Krebs and Sandow have shown that the algebra (1.12) exists, giving a representation for the matrices $D_{\alpha}$ and $X_{\alpha}$. This representation fulfils the second part of eq. (1.13) but not the first.

In order to compute the ground state wave-function one needs the "vacuum" expectation values of monomials of the form

$$
\left\langle 0\left|D_{\mu_{1}}^{r_{1}} D_{\mu_{2}}^{r_{2}} \cdots D_{\mu_{s}}^{r_{s}}\right| 0\right\rangle
$$

which are obtained from eqns (1.12) and (1.13). In order to find the wavefunction of the two-site problem one considers "vacuum" expectation values of monomials of degree two which are obtained from a system of linear equations. The solution of this system is not necessarily unique since the ground state might be degenerate. On completing this exercise the $L=3$ "vacuum" expectation values (1.16) are considered, and so on. This entire process, however, is more complicated than diagonalizing the Hamiltonian by brute force, and there should be a better way of solving the problem. This is indeed possible if the intensity matrices appearing in the Hamiltonian satisfy certain conditions. At this point, we shall mention only what is known in the $N=2$ case. In reference [14] Hinrichsen et al guessed a four-dimensional representation for the matrices $D_{0}, D_{1}, X_{0}$ and $X_{1}$ for a specific choice of intensity matrices and derived certain concentration profiles. In this specific case the problem can also be solved directly since the Hamiltonian can be written in terms of free fermions and it was this solution which inspired the guess. This reference is remarkable since it is the first place where the relations (1.12-16) appear.

We shall now describe the cases where the representation theory is understood. Consider the case where the only non-zero bulk rates are $\Gamma_{01}^{10}$ and 
$\Gamma_{10}^{01}$ but leave the surface (boundary) rates $L_{1}^{0}, L_{0}^{1}$ and $R_{1}^{0}, R_{0}^{1}$ arbitrary. This is the asymmetric exclusion model with open boundaries. One can choose $X_{1}=-X_{0}=1$ (the c-number 1) in equations (1.12) and (1.13) and after a linear transformation $D \rightarrow Y$ which includes constants related to the boundary rates, bring the relations to the form

$$
\left[Y_{0}, Y_{1}\right]=\sum_{\gamma, \delta \in\{0,1\}} c^{\gamma \delta} Y_{\gamma} Y_{\delta}+\sum_{\gamma \in\{0,1\}} c^{\gamma} Y_{\gamma}+c_{01}
$$

where $[A, B]=A B-B A$ and $c^{\gamma \delta}=c^{\delta \gamma}$. The parameters $c^{\gamma \delta}, c^{\gamma}$ and $c_{01}$ depend on the bulk and surface rates and we also obtain

$$
\left\langle 0\left|Y_{1}=0, \quad Y_{0}\right| 0\right\rangle=0, \quad\langle 0 \mid 0\rangle \neq 0 .
$$

The quadratic algebra with two generators (1.17) is well understood [13] and the Fock representations defined by eq. (1.18) are known [田, 9]. It was in the work of Derrida et al that Fock representations of quadratic algebras were used for the first time to find ground state wave-functions and to compute correlation functions. As one can notice from (1.17), (1.18), the "vacuum" expectation values of a monomial of degree $n$ in the $Y_{\alpha}$ 's is determined by the "vacuum" expectation values of a monomial of degree $n-1$ and one of degree $n-2$ in the $Y_{\alpha}$ 's. This implies the existence of recurrence relations between stationary distribution functions $P_{s}(\{\beta\})$ of lattices of different length $L$ (which is also the degree of the monomial in question). The first calculation of correlation functions in this model were performed using these recurrence relations without using the algebraic approach [2, 3].

One may try to take more bulk rates than the purely diffusive or hopping ones just described. The problem is that (1.12) with $X_{\alpha}$ 's chosen to be c-numbers gives three equations for the two generators $D_{0}$ and $D_{1}$, while one is enough to determine all "vacuum" expectation values as we have just seen. This implies relations between the rates. One trivial case corresponds to one-dimensional representations for the $D_{\alpha}$ (see [9]) when the (connected) correlation functions vanish. Even two-dimensional representations don't exist, since the relations on the rates obtained are incompatible with their positivity 15. Another possibility is to not restrict the $X_{\alpha}$ 's to be c-numbers, but (say) to take

$$
X_{\alpha}=A_{\alpha \gamma} D_{\gamma}+x_{\alpha}
$$

where $A_{\alpha \gamma}$ is an arbitrary $2 \times 2$ matrix and $x_{\alpha}$ 's are arbitrary c-numbers. Using the arbitrariness of the matrix elements $A_{\alpha \gamma}$, Krebs et al 15] have 
shown that it is possible to include more non-zero rates than just the hopping ones $\left(\Gamma_{10}^{01}\right.$ and $\left.\Gamma_{01}^{10}\right)$ and get a two-dimensional representation of the quadratic algebra, and hence obtain non-trivial correlation functions.

We would like to mention that the algebraic approach can be used not only in sequential processes described by the master equation, but also for parallel dynamics [16, 17, 18], where the algebraic problems to be solved are identical. Also, it is well worth recalling that matrix-product states first appeared in the literature for periodic boundary conditions in the work of Hakim and Nadal [19], and have also appeared in various publications by Fannes, Nachtergale and Werner [20] who also considered Bethe lattices. Parallel work was done by the Köln group [21]. The algebraic problem for periodic boundary conditions is very different since in this case, the wavefunction is given in terms of traces of monomials and not in terms of inner products in Fock states. Quadratic algebras can be used in this case too (see Refs. 22, 23, 24]).

In the present paper, we address the problem of stochastic processes involving $N \geq 2$ species. We shall consider, for the first time, not only the case of open boundary conditions, but also closed or mixed boundary conditions since the algebraic approach applies to these cases as well. In section 2, we first review some properties of intensity matrices which we shall use in the following sections. In section 3 , we consider the problem of the linear chain with closed ends (free boundary conditions). The boundary matrices $R$ and $L$ are identically zero and we shall consider solutions where $X_{\alpha}=0$. This case is not only interesting on its own, but it represents the natural first step before considering the chain with open ends where $R$ and $L$ are non-zero. (The choice $X_{\alpha}=0$ is compatible with eq. (1.13) and will take us to an interesting class of quadratic algebras.) This implies

$$
\Gamma_{\gamma \delta}^{\alpha \beta} D_{\alpha} D_{\beta}=0
$$

We shall call a quadratic algebra which contains only quadratic terms like in eq.(1.20) a polynomial algebra. (Obviously the algebra defined by eq.(1.17) is not one.) Equation (1.20) describes relations among monomials of degree two. The number of relations depends on the rank of the bulk intensity matrix and using eq. (1.14), we get the probability distribution for the twosites case. In order to have a solution for more sites, one needs consistency conditions which imply that the two ways of relating the cubic monomials 
$D_{\alpha} D_{\beta} D_{\gamma}$ and $D_{\gamma} D_{\beta} D_{\alpha}$ as depicted in the figure below

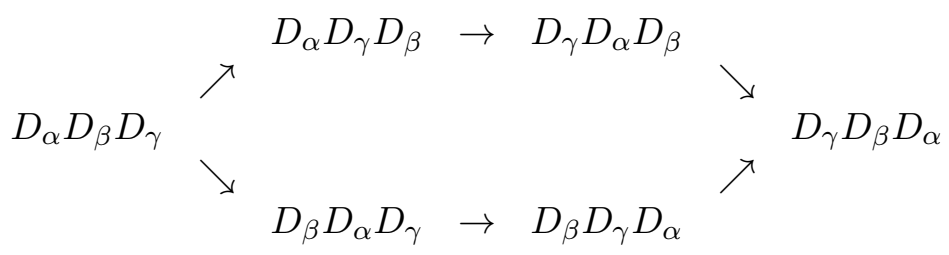

give the same result. The requirement of commutativity of the above diagram imposes constraints on the bulk rates and solves the $L=3$ case. In all the examples presented in the paper, we have checked that once the relations (1.21) are satisfied, no supplementary conditions on the rates arise from quartic (and in some cases) higher degree monomials. Similar conditions appear in quantum group structures [25, 26, 27] and it is no accident that various quantum planes or superplanes are solutions of the consistency conditions in some of the cases that we consider. Let us observe that for the closed chain case, one does not need to take average values $\langle 0|\cdots| 0\rangle$ in eq. (1.14); the ground state wave-functions (the ground state is often degenerate) and therefore the probablity distributions are the coefficients of the independent monomials. In section 3 we also answer the following question: if the ground state can be obtained by the present algebraic methods, is the Hamiltonian integrable? We shall show that the answer is in general, no.

In section 4 , we consider the case where the left end of the linear chain is open $\left(L_{\beta}^{\alpha} \neq 0\right)$ and the right end is closed $\left(R_{\beta}^{\alpha}=0\right)$. We again take $X_{\alpha}=0$ but now we need to add the condition:

$$
L_{\alpha}^{\beta}\langle 0| D_{\beta}=0 \quad(\alpha=0,1, . ., N-1)
$$

to equation (1.20), which comes from eq. (1.13). This implies new consistency conditions between the bulk and the left-boundary intensity matrices, which come from the equations

$$
L_{\alpha}^{\beta}\langle 0| D_{\beta} D_{\gamma}=0
$$

The consistency conditions depend on the rank of the $L$ matrix. Note that in this case, one has to drop the $|0\rangle$ symbol and retain the $\langle 0|$ symbol in equation (1.14), where the stationary probability distribution is expressed. Again there are no Fock representations in this case.

Fock representations appear in section 5, where we consider the linear chain with both ends open. This problem is much more difficult than the 
preceding ones. We therefore limit ourselves to the case $N=3$ only, since this case is complex enough and essentially different from the $N=2$ case. We also restrict our attention to the case of diffusion only (in the bulk), i.e. the only non-zero bulk rates are $\Gamma_{\beta \alpha}^{\alpha \beta}$. We look for representations for the case where the $X_{\alpha}$ 's are c-numbers. To our knowledge, only one example of this kind was known up to now [5]. It is possible to perform a linear transformation to bring (1.12) and (1.13) into the form

$$
\left[Y_{\alpha}, Y_{\beta}\right]=\sum_{\gamma, \delta \in\{0,1, \ldots, N-1\}} c_{\alpha \beta}^{\gamma \delta} Y_{\gamma} Y_{\delta}+\sum_{\gamma \in\{0,1, \ldots, N-1\}} c_{\alpha \beta}^{\gamma} Y_{\gamma}+c_{\alpha \beta},
$$

where

$$
c_{\alpha \beta}^{\gamma \delta}=c_{\alpha \beta}^{\delta \gamma}=-c_{\beta \alpha}^{\gamma \delta} ; \quad c_{\alpha \beta}^{\gamma}=-c_{\beta \alpha}^{\gamma} ; \quad c_{\alpha \beta}=-c_{\beta \alpha},
$$

with $N=3$, and the Fock conditions derived from eq.(1.13). We review the properties of the algebra (1.24) and stress that their properties are essentially different if $N>2$. We also show that the Fock conditions for $N>2$ are in general too numerous. This leads to a careful separation of cases depending on the rank of the boundary intensity matrices.

In section 6 , we show that if all the minors of the boundary matrices are non-zero, one can have representations of the quadratic algebra of dimension at most two with the bulk and boundary rates lying on some algebraic variety. In section 7 , we consider the case in which only one minor of $L_{\beta}^{\alpha}$ and one of $R_{\beta}^{\alpha}$ are non-zero. We give the representations of the algebra in this case. Other cases (three or more minors non-zero, for example) are not presented since the paper is long enough even without them. In Appendix A, we present simple physical processes where the formalism may be applied. (The results in the different sections of the paper may be used for several other applications.) We consider the problem of spontaneous symmetry breaking in a two species exclusion model with asymmetric diffusion proposed by Evans et al [5]. More boundary and bulk rates are considered than in the original model. This extension might allow a better understanding of the physics of the problem.

In section 8 , we consider the case in which all the principal cofactors are zero (the boundary intensity matrices have rank one). We are going to give four examples for this case.

The reader who might be tired of this long introduction is invited to go directly to section 9 , where a guide to finding the new results is presented along with a number of open questions. 


\section{Some properties of intensity matrices}

An $N \times N$ matrix $M$ whose elements $M_{i j}$ are such that the sum of all its entries on each column vanishes, i.e.

$$
\sum_{i=0}^{N-1} M_{i j}=0, \quad(j=0,1, \ldots, N-1),
$$

is called an I-matrix. I-matrices are closed under multiplication and form an algebra.

For stochastic problems, one considers I-matrices which have to satisfy the further restriction that the off-diagonal elements are real and non negative, since they are interpreted as probability rates of certain processes. Such an $N \times N$ matrix $M$ with real entries $M_{i j}$ is called an intensity matrix if

1. all its off-diagonal entries are non-negative, $M_{i j} \geq 0, i \neq j$,

2. and the diagonal elements are negative with

$$
M_{i i}=-\sum_{i \neq j} M_{i j}
$$

For any matrix $A$ with entries $A_{i j}$, if the Laplace row expansion of a determinant is written as $\operatorname{det} A \delta_{i k}=\sum_{j} A_{i j} \mathcal{A}_{k j}$ where $\mathcal{A}_{i j}$ is the cofactor corresponding to the $(i, j)^{t h}$ element of $A$. From this we can infer an important property of any zero-column sum matrices; namely their cofactors for each column are equal in magnitude, and in the case of intensity matrices, all the cofactors have the same sign. Let us denote the column- $j$ cofactor of an intensity matrix $M$ by the corresponding symbol in calligraphic font $\mathcal{M}_{j}$.

It therefore follows that a system of linear equations

$$
\sum_{n=0}^{N-1} M_{m n} x_{n}=0
$$

has a solution given by

$$
x_{n}=\xi \mathcal{M}_{n}
$$

for any constant (independent of $n$ ) $\xi$ if the rank of $M$ is $N-1$. 
Consider two intensity matrices $F$ and $G$, and their sum

$$
E=F+G
$$

which is also an intensity matrix. The following identity is true for $N=1,2$ and 3 only:

$$
\sum_{m=0}^{N-1}\left(F_{n m}-G_{n m}\right) \mathcal{E}_{m}=(N-1) \sum_{m=0}^{N-1}\left(F_{n m} \mathcal{G}_{m}-G_{n m} \mathcal{F}_{m}\right),
$$

where as noted earlier, the calligraphic $\mathcal{E}, \mathcal{F}, \mathcal{G}$ with subscript $m$ denotes the cofactors of $E, F, G$ for the $m^{t h}$ column. The last identity is useful for the 3 species case. Since we are going to use them often in the next few sections, we give the expressions for the three distinct cofactors of a $3 \times 3$ intensity matrix $F$ with matrix elements $F_{n}^{m}$ :

$$
\begin{aligned}
& \mathcal{F}_{0}=F_{0}^{1} F_{1}^{2}+F_{0}^{2} F_{0}^{1}+F_{0}^{2} F_{2}^{1}, \\
& \mathcal{F}_{1}=F_{1}^{0} F_{0}^{2}+F_{1}^{2} F_{1}^{0}+F_{1}^{2} F_{2}^{0}, \\
& \mathcal{F}_{2}=F_{2}^{0} F_{0}^{1}+F_{2}^{1} F_{2}^{0}+F_{2}^{1} F_{1}^{0} .
\end{aligned}
$$

\section{$3 \quad$ Steady states for a linear chain with closed ends (Ground states for quantum spin chains with free boundary conditions)}

As mentioned in the introduction, we consider ground state wave-functions of the form

$$
\left.\left|P_{s}\right\rangle\right\rangle=\prod_{k=1}^{L}\left(\sum_{\mu_{k}=0}^{N-1} D_{\mu_{k}} u_{\mu_{k}}\right),
$$

where the matrices $D_{\mu}$ satisfy equations (1.20) and the consistency conditions (1.21). The type of wave-functions we get depends on the rank of the bulk intensity matrix $\Gamma_{\gamma \delta}^{\alpha \beta}$. Let us first consider the case where the rank is the maximum possible, i.e. $N^{2}-1$, and all principal minors are non-zero. We denote by $\mathcal{G}_{\alpha \beta}$ the cofactors of $\Gamma_{\gamma \delta}^{\alpha \beta}$ (see section 2). Using equation (2.4) we get

$$
D_{\alpha} D_{\beta}=\frac{\mathcal{G}_{\alpha \beta}}{\mathcal{G}_{\gamma \delta}} D_{\gamma} D_{\delta}
$$


There are two ways in which the cubic monomial $D_{\alpha} D_{\beta} D_{\gamma}$ can be related to $D_{\mu} D_{\rho} D_{\sigma}$ :

$$
\begin{aligned}
& \text { (i) } D_{\alpha} D_{\beta} D_{\gamma}=\frac{\mathcal{G}_{\alpha \beta}}{\mathcal{G}_{\mu \nu}} D_{\mu} D_{\nu} D_{\gamma}=\frac{\mathcal{G}_{\alpha \beta}}{\mathcal{G}_{\mu \nu}} \frac{\mathcal{G}_{\nu \gamma}}{\mathcal{G}_{\rho \sigma}} D_{\mu} D_{\rho} D_{\sigma} \\
& \text { (ii) } D_{\alpha} D_{\beta} D_{\gamma}=\frac{\mathcal{G}_{\beta \gamma}}{\mathcal{G}_{\nu \sigma}} D_{\alpha} D_{\nu} D_{\sigma}=\frac{\mathcal{G}_{\beta \gamma}}{\mathcal{G}_{\nu \sigma}} \frac{\mathcal{G}_{\alpha \nu}}{\mathcal{G}_{\mu \rho}} D_{\mu} D_{\rho} D_{\sigma}
\end{aligned}
$$

Comparing (i) and (ii) in equation (3.3) (and setting $\rho=\nu$ ) we get:

$$
\mathcal{G}_{\alpha \beta} \mathcal{G}_{\nu \gamma}=\mathcal{G}_{\alpha \nu} \mathcal{G}_{\beta \gamma}
$$

and hence the condition on the cofactors (on setting $\nu=\alpha$ and $\beta=\gamma$ ) is

$$
\mathcal{G}_{\alpha \beta}= \pm \sqrt{\mathcal{G}_{\alpha \alpha} \mathcal{G}_{\beta \beta}}=\mathcal{G}_{\beta \alpha} .
$$

The $+(-)$ is taken if $N$ is odd (even). This implies that the $D_{\alpha}$ 's can be taken as c-numbers:

$$
D_{\alpha}=\xi \sqrt{\mathcal{G}_{\alpha \alpha}}
$$

where $\xi$ is a constant. The ground state is unique and the (connected) correlation functions manifestly zero.

The whole structure of the possible solutions for the ground state wavefunction depends on the rank of the bulk intensity matrix $\Gamma_{\gamma \delta}^{\alpha \beta}$. We shall restrict ourselves to the cases $N=2,3$. Before proceeding further, we would like to consider some questions about integrability. We are going to find that for some intensity matrices we can find ground-state wave-functions. In general the ground is degenerate, which might imply the existence of some symmetry in the Hamiltonian. What is this symmetry? On the other hand, one can also ask the (possibly related) question, if the ground states are obtained from polynomial algebras, is the Hamiltonian integrable? In general, the answer is negative. Exact integrability requires more than the exact expression for the ground-state wavefunction.

In certain cases, the $e_{k}$ defined by

$$
e_{k}:=H_{k, k+1},
$$

where $H_{k, k+1}$ is the Hamiltonian density given in equation (1.9), satisfy the relations of a Hecke algebra:

$$
\begin{aligned}
e_{k} e_{k \pm 1} e_{k}-e_{k} & =e_{k \pm 1} e_{k} e_{k \pm 1}-e_{k \pm 1} \\
{\left[e_{k}, e_{j}\right] } & =0, \text { for }|k-j| \geq 2 \\
e_{k}^{2} & =\left(q+q^{-1}\right) e_{k}
\end{aligned}
$$


In this case, one can find a spectral parameter dependent solution $\check{R}(u)$ of the Yang-Baxter equation via "Baxterization" [28, 29]. One can check that the generators $e_{k}$ satisfy further relations among themselves which define various quotients of the Hecke algebra [30]. For our purposes, it is sufficient to state that the $H_{k, k+1}$ belong to such quotients that are classified by a pair of natural numbers $(P, M)$. For each quotient labelled by $(P, M)$ the Hamiltonian whose density is given by the appropriate $e_{k}$ defines a spin chain [31] with $U_{q} s u(P \mid M)$ (which is the Schur-Weyl dual of the $(P, M)$ Hecke quotient) as its symmetry algebra. (For further details, see [10].)

A more general criterion for integrability at the level of Hamiltonian densities was introduced by Reshetikhin 32 for the case of $R$-matrices whose dependence on the spectral parameter is of difference type. By expanding the $R$ and row transfer matrices in powers of the spectral parameter, it was shown to be necessary that the Hamiltonian densities $e_{k}$ satisfy the following relation

$$
\left[e_{k}+e_{k+1},\left[e_{k}, e_{k+1}\right]\right]=W_{k}-W_{k+1}
$$

where $W_{k}$ is a tensor product of identity matrices at all sites on the chain and an arbitrary matrix at the $k^{t h}$ site.

$\bullet \underline{N=2}$

We now consider the $N=2$ case. We shall on occasion refer to the state "1" as that denoting the presence of a particle and the state "0" to its absence. If all the minors are non-zero, and the intensity matrix is of maximal possible rank (i.e. 3) equation (3.6) applies. We consider the cases where the rank is less than 3 . Let us first consider the case

$$
\Gamma_{10}^{00}=\Gamma_{01}^{00}=\Gamma_{11}^{00}=\Gamma_{00}^{01}=\Gamma_{00}^{10}=\Gamma_{00}^{11}=0,
$$

where the bulk intensity matrix effectively reduces (from a $4 \times 4$ ) to a $3 \times 3$ matrix with rows and columns labelled by $(1,0),(0,1)$ and $(1,1)$. Let $\mathcal{G}_{1,0}^{\prime}$, $\mathcal{G}_{0,1}^{\prime}$ and $\mathcal{G}_{1,1}^{\prime}$ be the cofactors of this new intensity matrix. In order to have a polynomial algebra we must have the condition

$$
\mathcal{G}_{1,0}^{\prime}=\mathcal{G}_{0,1}^{\prime}
$$

and the algebra is

$$
D_{1} D_{0}=D_{0} D_{1}=\frac{\mathcal{G}_{1,0}^{\prime}}{\mathcal{G}_{1,1}^{\prime}} D_{1}^{2}
$$

The ground state for $L$ sites is now doubly degenerate. One state corresponds to $D_{0}^{L}$ and the other contains all the monomials which have at least one $D_{1}$, and can be brought by eq.(3.12) to be proportional to $D_{1}^{L}$. 
Next we consider the situation where we have only diffusion of particles and their annihilation if two particles are on neighbouring sites. This is attained by choosing the following rates to vanish (the rest being non-zero):

$$
\Gamma_{10}^{00}=\Gamma_{01}^{00}=\Gamma_{11}^{00}=\Gamma_{00}^{01}=\Gamma_{00}^{10}=\Gamma_{11}^{01}=\Gamma_{11}^{10}=0 .
$$

In this case the algebra is

$$
D_{0} D_{1}=q^{2} D_{1} D_{0} ; \quad D_{1}^{2}=0
$$

where

$$
q=\sqrt{\frac{\Gamma_{01}^{10}}{\Gamma_{10}^{01}}} .
$$

If we choose the time scale such that $\Gamma_{01}^{10} \Gamma_{10}^{01}=1$, the set of $H_{k, k+1}$ satisfies the relations for the $(1,1)$ quotient of the Hecke algebras centralizing $U_{q}(s u(1 \mid 1))$. The ground state for $L$ sites is doubly degenerate corresponding to the words $D_{0}^{L}$ and $D_{1} D_{0}^{L-1}$.

Yet another example of an intensity matrix which gives rise to a quadratic algebra is

$$
\left(\begin{array}{cccc}
-\Gamma_{10}^{00} & 0 & \Gamma_{00}^{10} & 0 \\
0 & -\Gamma_{11}^{01} & 0 & \Gamma_{01}^{11} \\
\Gamma_{10}^{00} & 0 & -\Gamma_{00}^{10} & 0 \\
0 & \Gamma_{11}^{01} & 0 & -\Gamma_{01}^{11}
\end{array}\right)
$$

which gives the following quadratic algebra:

$$
D_{0} D_{1}=\frac{1}{q^{2}} D_{1}^{2} \quad \text { and } \quad D_{1} D_{0}=q^{2} D_{0}^{2}, \quad \text { where } \quad q^{2}=\frac{\Gamma_{10}^{00}}{\Gamma_{00}^{10}}=\frac{\Gamma_{11}^{01}}{\Gamma_{01}^{11}}
$$

after solving for the associativity constraints coming from the cubic terms.

Yet another possibility is to set more rates to 0 , in addition to those which led to equation (3.14). Upon setting $\Gamma_{00}^{11}, \Gamma_{01}^{11}$ and $\Gamma_{10}^{11}$ to zero as well, which means we are only considering diffusion, we are led to

$$
D_{0} D_{1}=\frac{\Gamma_{01}^{10}}{\Gamma_{10}^{01}} D_{1} D_{0}
$$

The ground state is now $(L+1)$ times degenerate - each wave-function has a given number of $D_{1}$ 's and the coefficients of the tensor products of the $u_{k}^{(\mu)}$ 's in eq.(3.1) correspond to the $q$-deformation of the symmetrizer 
corresponding to the Young diagram with one row and $L$ boxes [10], with $q=\sqrt{\Gamma_{01}^{10} / \Gamma_{10}^{01}}$. The quadratic relation

$$
D_{0} D_{1}=0
$$

corresponds not only to the case where $\Gamma_{10}^{01}$ is the only non-zero rate, but more generally $\Gamma_{\alpha \beta}^{01}$ is non-zero for all $\alpha, \beta$. Again the ground state is $(L+1)$ times degenerate. Since our intention is to generalize the problem to $N>2$, it is useful to present visually the non-zero matrix elements which correspond to the different quadratic relations. The rows and columns below are labelled $(0,0),(0,1),(1,0)$ and $(1,1)$ consecutively.

$$
\begin{aligned}
& \left(\begin{array}{llll}
\square & \square & \square & \square \\
\square & \square & \square & \square \\
\square & \square & \square & \square \\
\square & \square & \square & \square
\end{array}\right) \quad \text { eq.(3.6) } \quad\left(\begin{array}{cccc}
\cdot & \cdot & \cdot & \cdot \\
\cdot & \square & \square & \square \\
\cdot & \square & \square & \square \\
\cdot & \square & \square & \square
\end{array}\right) \quad \text { eq.(3.12) } \\
& \left(\begin{array}{cccc}
\cdot & \cdot & \cdot & \square \\
\cdot & \square & \square & \square \\
\cdot & \square & \square & \square \\
\cdot & \cdot & \cdot & \square
\end{array}\right) \text { eq.(3.14) } \quad\left(\begin{array}{cccc}
\square & \cdot & \square & \cdot \\
\cdot & \square & \cdot & \square \\
\square & \cdot & \square & \cdot \\
\cdot & \square & \cdot & \square
\end{array}\right) \quad \text { eq.(3.17) } \\
& \left(\begin{array}{cccc}
\cdot & \cdot & \cdot & \cdot \\
\cdot & \square & \square & \cdot \\
\cdot & \square & \square & \cdot \\
\cdot & \cdot & \cdot & \cdot
\end{array}\right) \text { eq.(3.18) } \quad\left(\begin{array}{cccc}
\cdot & \square & \cdot & \cdot \\
\cdot & \square & \cdot & \cdot \\
\cdot & \square & \cdot & \cdot \\
\cdot & \square & \cdot & \cdot
\end{array}\right)
\end{aligned}
$$

We could not obtain algebras with non-scalar $D_{0}$ and $D_{1}$ for any other choice of locations in the intensity matrix for the non-zero rates than those depicted above.

$\bullet \underline{N=3}$

We now consider the $N=3$ case. We are not going to list all the possible quadratic relations for the two-site problem which are compatible with the 3 -site problem (i.e. the cubic relations are compatible with the associative application of the quadratic relations) but list a few example which do. We shall, in some cases, interpret the state variables $1,2,0$ as those of two species of particles and holes respectively. First, we consider the case in which the 
two-site bulk intensity matrix decouples into three intensity matrices (the other rates being zero):

$$
\left(\begin{array}{cc}
-\Gamma_{10}^{01} & \Gamma_{01}^{10} \\
\Gamma_{10}^{01} & -\Gamma_{01}^{10}
\end{array}\right) \quad\left(\begin{array}{cc}
-\Gamma_{20}^{02} & \Gamma_{02}^{20} \\
\Gamma_{20}^{02} & -\Gamma_{02}^{20}
\end{array}\right)
$$

and

$$
\left(\begin{array}{ccc}
-\left(\Gamma_{21}^{00}+\Gamma_{12}^{00}\right) & \Gamma_{00}^{12} & \Gamma_{00}^{21} \\
\Gamma_{12}^{00} & -\left(\Gamma_{00}^{12}+\Gamma_{21}^{12}\right) & \Gamma_{12}^{21} \\
\Gamma_{21}^{00} & \Gamma_{21}^{12} & -\left(\Gamma_{00}^{21}+\Gamma_{12}^{21}\right)
\end{array}\right)
$$

We denote by $\mathcal{J}_{00}, \mathcal{J}_{12}$ and $\mathcal{J}_{21}$ the cofactors of the $3 \times 3$ intensity matrix. The quadratic relations obtained are

$$
\begin{array}{cc}
D_{0} D_{1}=\gamma D_{1} D_{0} & D_{2} D_{0}=\delta D_{0} D_{2}, \\
D_{1} D_{2}=\beta D_{0}^{2} & \alpha D_{2} D_{1}=\beta D_{0}^{2} .
\end{array}
$$

The cubic relations obtained by considering words in the $D$ 's of degree 3 are compatible with the quadratic relations provided

$$
\alpha=\frac{\mathcal{J}_{12}}{\mathcal{J}_{21}}=\gamma^{-2} ; \quad \beta=\frac{\mathcal{J}_{12}}{\mathcal{J}_{00}} ; \quad \gamma=\delta=\frac{\Gamma_{01}^{10}}{\Gamma_{10}^{01}}=\frac{\Gamma_{20}^{02}}{\Gamma_{02}^{20}} .
$$

There are $(2 L+1)$ wave-functions with energy eigenvalue zero. They are given in terms of the monomials

$$
D_{0}^{L-m} D_{1}^{m}, \quad D_{0}^{L-n} D_{2}^{n}
$$

This gives an explanation as to why it was possible to calculate the probability distribution exactly in 33] for the particular case where interchange of particles is forbidden $\left(\Gamma_{21}^{12}=\Gamma_{12}^{21}=0\right)$. Interpreting the matrix entries in eq. (3.20) as transition rates involving two species of particles 1 and 2 we notice that the difference in the number of particles of types 1 and 2 is conserved under this stochastic dynamics.

We now consider the intensity matrix of the block diagonal form

$$
\left(\begin{array}{ccccccccc}
\Gamma_{00}^{00} & \Gamma_{00}^{01} & \Gamma_{00}^{02} & 0 & 0 & 0 & 0 & 0 & 0 \\
\Gamma_{01}^{00} & \Gamma_{01}^{01} & \Gamma_{01}^{02} & 0 & 0 & 0 & 0 & 0 & 0 \\
\Gamma_{02}^{00} & \Gamma_{02}^{01} & \Gamma_{02}^{02} & 0 & 0 & 0 & 0 & 0 & 0 \\
0 & 0 & 0 & \Gamma_{10}^{10} & \Gamma_{10}^{11} & \Gamma_{10}^{12} & 0 & 0 & 0 \\
0 & 0 & 0 & \Gamma_{11}^{10} & \Gamma_{11}^{11} & \Gamma_{11}^{12} & 0 & 0 & 0 \\
0 & 0 & 0 & \Gamma_{12}^{10} & \Gamma_{12}^{11} & \Gamma_{12}^{12} & 0 & 0 & 0 \\
0 & 0 & 0 & 0 & 0 & 0 & \Gamma_{20}^{20} & \Gamma_{20}^{21} & \Gamma_{20}^{22} \\
0 & 0 & 0 & 0 & 0 & 0 & \Gamma_{21}^{20} & \Gamma_{21}^{21} & \Gamma_{21}^{22} \\
0 & 0 & 0 & 0 & 0 & 0 & \Gamma_{22}^{20} & \Gamma_{22}^{21} & \Gamma_{22}^{22}
\end{array}\right),
$$


and we denote the cofactors of the $3 \times 3$ block containing $\Gamma_{i i}^{i i}$ by $\mathcal{G}_{(i)}^{\alpha}$, where $\alpha=0,1,2$ labels the $(\alpha+1)^{t h}$ column within each block. The algebra is defined by the six relations

$$
D_{\alpha} D_{\beta}=\frac{f_{\beta}}{f_{\alpha}} D_{\alpha}^{2}
$$

where

$$
\begin{gathered}
\frac{f_{1}}{f_{0}}=\frac{\mathcal{G}_{(0)}^{1}}{\mathcal{G}_{(0)}^{0}}=\frac{\mathcal{G}_{(1)}^{1}}{\mathcal{G}_{(1)}^{0}} ; \quad \frac{f_{0}}{f_{2}}=\frac{\mathcal{G}_{(2)}^{0}}{\mathcal{G}_{(2)}^{2}}=\frac{\mathcal{G}_{(0)}^{0}}{\mathcal{G}_{(2)}^{0}} ; \\
\frac{f_{2}}{f_{1}}=\frac{\mathcal{G}_{(1)}^{2}}{\mathcal{G}_{(1)}^{1}}=\frac{\mathcal{G}_{(2)}^{2}}{\mathcal{G}_{(2)}^{1}} ; \quad \mathcal{G}_{(0)}^{0} \mathcal{G}_{(1)}^{1} \mathcal{G}_{(2)}^{2}=\mathcal{G}_{(0)}^{1} \mathcal{G}_{(1)}^{2} \mathcal{G}_{(2)}^{0} .
\end{gathered}
$$

The ground state is 3-fold degenerate, the independent words being $D_{0}^{L}, D_{1}^{L}$ and $D_{2}^{L}$. This degeneracy arises since the type of particle located at the first site $(0,1,2)$ is unchanged by the stochastic dynamics.

An interesting example with 4 quadratic relations is

$$
\begin{array}{ccc}
\Gamma_{10}^{01} D_{0} D_{1} & =\Gamma_{01}^{10} D_{1} D_{0} \\
\Gamma_{21}^{12} D_{1} D_{2} & =\Gamma_{12}^{21} D_{2} D_{1} \\
\Gamma_{02}^{20} D_{2} D_{0} & = & \Gamma_{20}^{02} D_{0} D_{2} \\
D_{1}^{2} & = & 0 .
\end{array}
$$

These relations are obtained if the only non-zero rates are those that appear in (3.28) and $\Gamma_{\alpha \beta}^{11},(\alpha, \beta \in\{0,1\})$. In order to find the degeneracy of the ground state, notice that if we set

$$
\Gamma_{\beta \alpha}^{\alpha \beta}=\frac{1}{\Gamma_{\alpha \beta}^{\beta \alpha}},(\alpha \neq \beta) \quad \text { and } \quad \frac{\Gamma_{10}^{01}}{\Gamma_{01}^{10}}=\frac{\Gamma_{20}^{02}}{\Gamma_{02}^{20}}=\frac{\Gamma_{21}^{12}}{\Gamma_{12}^{21}}=q^{2}
$$

we obtain the $(2,1)$ quotient of the Hecke algebra [10], and by Schur-Weyl duality, the Hamiltonian is $U_{q}(s u(2 \mid 1))$ symmetric. The ground state wavefunctions are proportional to the monomials symmetrized using the relations $(3.28,29)$ and are therefore given by the representation of the superalgebra which corresponds to a Young diagram with $L$ boxes in one row 34].

A physically important example is the case in which we consider hopping and interchange of particles; we set the rates $\Gamma_{\alpha \beta}^{11}$ to zero in the previous example to get the three relations

$$
D_{0} D_{1}=\frac{\Gamma_{01}^{10}}{\Gamma_{10}^{01}} D_{1} D_{0}, D_{1} D_{2}=\frac{\Gamma_{12}^{21}}{\Gamma_{21}^{12}} D_{2} D_{1}, D_{2} D_{0}=\frac{\Gamma_{20}^{02}}{\Gamma_{02}^{20}} D_{0} D_{2} .
$$


We have checked whether the Hamiltonian thus defined satisfies the necessary condition as prescribled by Reshetikhin (eq. (3.9)) for being integrable. We found that in order to be so, the rates must satisfy the relations (3.29) and we get the $(3,0)$ quotient of the Hecke algebra and the Hamiltonian is $U_{q}(s u(3))$ symmetric. This result is important because it shows that although the ground state of the Hamiltonian can be obtained by algebraic means, the spectrum of the Hamiltonian cannot, unless supplementary conditions are satisfied. We have also checked other cases with the same result. If the relations (3.29) are satisfied, the ground states again give the representation of $U_{q}(s u(3))$ which correspond to the Young diagram with $L$ boxes in one row [34].

We can also consider the case where the only rates that are non-zero are $\Gamma_{10}^{01}, \Gamma_{01}^{10}, \Gamma_{20}^{02}$ and $\Gamma_{02}^{20}$. The only two relations that survive are

$$
\Gamma_{10}^{01} D_{0} D_{1}=\Gamma_{01}^{10} D_{1} D_{0} \quad \text { and } \quad \Gamma_{20}^{02} D_{0} D_{2}=\Gamma_{02}^{20} D_{2} D_{0},
$$

and the degeneracies are huge since any sequence of the state indices 1 and 2 is invariant under the dynamics and can be computed using the results of ref. 35 .

Finally, one can have only one relation

$$
D_{i} D_{j}=0
$$

if the only non-zero rates are $\Gamma_{\alpha, \beta}^{i, j}$ for any $\alpha, \beta$.

Since we have only looked at particular cases, it is most likely that we have not exhausted all possible quadratic algebras arising from $9 \times 9$ intensity matrices. We have not been able to find other examples which give nontrivial (non-scalar) representations for the relations emerging from a choice of intensity matrix. The rules of this game are however very simple and can be applied by the reader to find other examples. In the $9 \times 9$ intensity matrix, one takes several diagonal blocks of smaller intensity matrices with non-zero rates and columns of possibly non-zero rates of the form depicted below. 


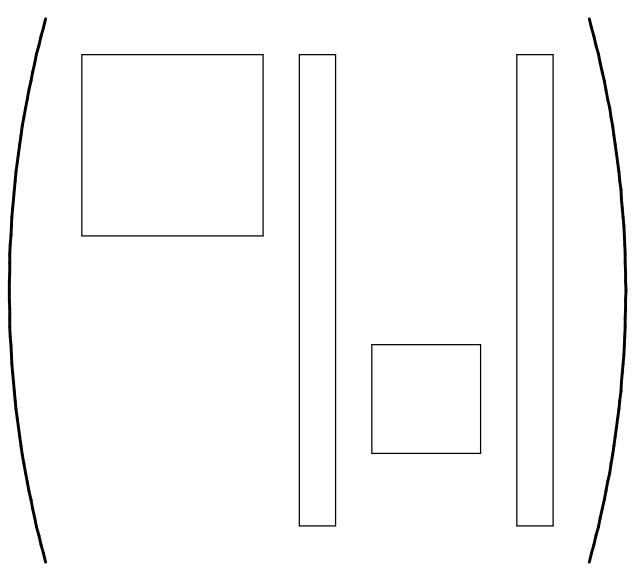

Notice that there are no rectangular boxes which span entire rows of the matrix $\Gamma$ above.

This intensity matrix $\Gamma$ gives quadratic relations which then have to be checked for consistency for the 3 -site case. We can do the same with another intensity matrix $\Gamma^{\prime}:=P_{\mu \nu} \Gamma P_{\mu \nu}$, where $P_{\mu \nu}$ is a permutation matrix,

$$
P_{\mu \nu}\left(u_{\mu_{k}} \otimes u_{\nu_{k+1}}\right)=\left(u_{\nu_{k}} \otimes u_{\mu_{k+1}}\right)
$$

where $u_{\mu_{k}, \nu_{k}}$ are defined before eq.(1.15). The same procedure applies for any number of species $N$.

To sum up, we have shown that there are a number of quadratic algebras which allow the computation of the ground state wave-function. What is missing is a complete classification of the algebras. What is also missing is an understanding of the origin of the degeneracies. Are the ground state wavefunctions some representations of some algebra? In the special case where the Hamiltonian densities are generators of a Hecke algebra, the answer is known, but not in the general case.

We would also like to emphasize that the ground state wave-functions thus computed are non-trivial in the sense that connected correlations functions in these states are non-zero. One simple example is already known. This is given in eq. (3.18) [36]. The method used in [36] to compute the correlation functions was different. Other cases can be considered using the results of this section. 


\section{Linear chain with left end open and right end closed}

As discussed in section 1, the ground state wave-function is given by the expression

$$
\left.\left|P_{s}\right\rangle\right\rangle=\langle 0| \prod_{k=1}^{L}\left(\sum_{\mu_{k}=0}^{N-1} D_{\mu_{k}} u_{\mu_{k}}\right),
$$

where the $D_{\mu}$ 's satisfy one of the polynomial algebras described in the last section and a new condition (see eq. (1.22))

$$
\sum_{\beta=0}^{N-1} L_{\alpha}^{\beta}\langle 0| D_{\beta}=0,
$$

where $L_{\alpha}^{\beta}$ is an intensity matrix with cofactors $\mathcal{L}_{\beta}$. We consider the cases $N=2$ and $N=3$ only. The following conditions on the $D$ matrices are obtained depending on the structure of the boundary intensity matrix. In each case, only the non-zero rates are written down.

$\underline{N=2}$

1.

$$
\langle 0| D_{1}=\frac{L_{1}^{0}}{L_{0}^{1}}\langle 0| D_{0} \quad\left(L_{0}^{1}, L_{1}^{0} \neq 0\right)
$$

2.

$$
\langle 0| D_{1}=0 \quad\left(L_{0}^{1} \neq 0\right)
$$

3.

$$
\langle 0| D_{0}=0 \quad\left(L_{1}^{0} \neq 0\right)
$$

$\underline{N=3}$

For the case where at least one principal cofactor, e. g. $\mathcal{L}_{0}$, is non-zero

$$
\langle 0| D_{\alpha}=\frac{\mathcal{L}_{\alpha}}{\mathcal{L}_{0}}\langle 0| D_{0} \quad(\alpha=1,2)
$$

and one obtains two constraints. If all the principal cofactors are 0, only two rates are non-zero. We can distinguish the following cases: 
1.

$$
\langle 0| D_{1}=\frac{L_{1}^{0}}{L_{0}^{1}}\langle 0| D_{0} \quad L_{0}^{1}, L_{1}^{0} \neq 0
$$

2 .

$$
\langle 0| D_{2}=\frac{L_{2}^{0}}{L_{0}^{2}}\langle 0| D_{0} \quad L_{0}^{2}, L_{2}^{0} \neq 0
$$

3.

$$
\langle 0| D_{2}=\frac{L_{2}^{1}}{L_{1}^{2}}\langle 0| D_{1} \quad L_{2}^{1}, L_{1}^{2} \neq 0
$$

4.

$$
\langle 0| D_{1}=0 \quad\left(L_{0}^{1}+L_{2}^{1}\right) \neq 0 ;
$$

5.

$$
\langle 0| D_{0}=0 \quad\left(L_{1}^{0}+L_{2}^{0}\right) \neq 0 ;
$$

6.

$$
\langle 0| D_{2}=0 \quad\left(L_{0}^{2}+L_{1}^{2}\right) \neq 0 .
$$

Notice that for the last six cases, one has only one constraint. The conditions (4.3) to (4.12) have to be compatible with the polynomial algebras. In this way one can, in some cases, find relations between the bulk rates only. In most cases the degeneracy of the ground state is lifted.

The marriage of the bulk algebra with the boundary conditions (4.312 ) is a straightforward mathematical exercise. Therefore, we shall confine ourselves to models of asymmetric diffusion where the only non-zero rates are $\Gamma_{\beta \alpha}^{\alpha \beta}$ and give examples of how the concentration profiles can be computed. These are the cases for which the relevant algebras are given in eq. (3.18) for $N=2$ and eq. (3.30) for $N=3$.

We shall start with the $N=2$ case. We have the algebra

$$
D_{0} D_{1}=q^{2} D_{1} D_{0}
$$

and one of the conditions (4.3-5). The conditions eq. (4.4) and (4.5) give a trivial ground state: $\langle 0| D_{0}^{L}$ (eq. 4.4 ) or $\langle 0| D_{1}^{L}$ (eq. 4.5 ). We shall rewrite eq. (4.3) as follows:

$$
\langle 0| D_{1}=\mu\langle 0| D_{0} .
$$

There are no constraints connecting $q^{2}$ and $\mu$. Assume that $D_{1}$ indicates the presence of a particle on any particular site and $D_{0}$ the lack of one, i.e. 
holes. The concentration of particles at a distance $k$ from the left boundary for a chain of length $L$ is [4]

$$
c(k)=\frac{1}{Z_{L}}\langle 0| C^{k-1} D_{1} C^{L-k},
$$

where

$$
Z_{L}=\langle 0| C^{L}, \quad C=D_{0}+D_{1} .
$$

A straightforward calculation gives

$$
c(k)=\frac{1}{1+\frac{1}{\mu} q^{2(1-k)}} .
$$

The connected two-point correlation function

$$
c(k, l)=\frac{1}{Z_{L}}\langle 0|\left(C^{k-1} D_{1} C^{l-k-1} D_{1} C^{L-l}\right)-c(k) c(l)
$$

vanishes.

An interesting case occurs for the algebra

$$
D_{1} D_{0}=0
$$

which corresponds to $\Gamma_{10}^{01}=0$ and consider the boundary condition $L_{1}^{0}=0$ (eq. 4.4). Upon inserting these conditions into eq. (4.15) we obtain

$$
c(k)=\frac{\langle 0| \sum_{j=1}^{k-1} D_{0}^{j} D_{1}^{L-j}}{\langle 0| \sum_{j=1}^{L} D_{0}^{j} D_{1}^{L-j}}
$$

and we cannot go any further because our algebraic rules do not allow us to evaluate this expression. We leave it as an exercise to the reader to find the physical reasons why the method appears not to work in this case, and to do the calculation properly.

We now consider the $N=3$ states problem and illustrate the method in the case of eq. (3.30), which we rewrite for convenience:

$$
q_{0} D_{1} D_{2}=D_{2} D_{1} . \quad q_{1} D_{2} D_{0}=D_{0} D_{2}, \quad q_{2} D_{0} D_{1}=D_{1} D_{0},
$$

where

$$
q_{0}=\frac{\Gamma_{21}^{12}}{\Gamma_{12}^{21}}, \quad q_{1}=\frac{\Gamma_{02}^{20}}{\Gamma_{20}^{02}}, \quad q_{2}=\frac{\Gamma_{10}^{01}}{\Gamma_{01}^{10}} .
$$


We consider the boundary conditions (4.6) and assume all the rates in (4.22) to be non-zero. Setting $\mu_{\alpha}:=\mathcal{L}_{\alpha} / \mathcal{L}_{0}$, we have

$$
\langle 0| D_{1}=\mu_{1}\langle 0| D_{0} \quad \text { and } \quad\langle 0| D_{2}=\mu_{2}\langle 0| D_{0} .
$$

As we shall now show, consistency conditions on the rates show up. Let us first compute

$$
\begin{array}{ccc}
q_{0}\langle 0| D_{1} D_{2} & =q_{0} \mu_{1}\langle 0| D_{0} D_{2}=q_{0} q_{1} \mu_{1}\langle 0| D_{2} D_{0}=q_{0} q_{1} \mu_{1} \mu_{2}\langle 0| D_{0}^{2} \\
\langle 0| D_{2} D_{1} & =\mu_{2}\langle 0| D_{0} D_{1}=\left(\mu_{2} / q_{2}\right)\langle 0| D_{1} D_{0}=\left(\mu_{1} \mu_{2} / q_{2}\right)\langle 0| D_{0}^{2}
\end{array}
$$

From the first equation in (4.21) and equations (4.24) we get

$$
q_{0} q_{1} q_{2}=1
$$

Note that this condition constrains only the bulk rates. The concentration profiles for particles " 1 " and "2" ("0" denotes vacancies) can easily be computed [5] as

$$
\begin{gathered}
c_{1}(k)=\frac{1}{Z_{L}}\langle 0| C^{k-1} D_{1} C^{L-k} ; \quad c_{2}(k)=\frac{1}{Z_{L}}\langle 0| C^{k-1} D_{2} C^{L-k} \\
Z_{L}=\langle 0| C^{L} ; \quad C=D_{0}+D_{1}+D_{2} .
\end{gathered}
$$

Using equations (4.21), (4.23) and (4.25) we obtain

$$
c_{1}(k)=\frac{\mu_{1} q_{2}^{1-k}}{1+\mu_{1} q_{2}^{1-k}+\mu_{2} q_{1}^{k-1}} ; \quad \frac{c_{1}(k)}{c_{2}(k)}=\frac{\mu_{1}}{\mu_{2}} q_{0}^{k-1} .
$$

We are not aware of the existence of another method which would give us the expressions (4.27). The connected two-point correlation function is zero.

\section{The open chain three-state exclusion models}

This is a much more difficult problem. We limit our investigation to exclusion models in which the only non-vanishing rates in the bulk are

$$
g_{\alpha \beta}:=\Gamma_{\beta \alpha}^{\alpha \beta}
$$

and make the assumption that the matrices $X_{\mu}$ 's in equation (1.12) are cnumbers, which we shall call $x_{\mu}$. As we shall see below, the choice $x_{\mu}=0$ 
is possible for pathological choices of boundary conditions only. The choice (5.1) is not only justified by the interest in these physical processes but as shown later, (see the discussion after eq. (5.24)) in this way one obtains quadratic algebras which are understood [12, 13].

We are looking therefore at Fock representations of the algebra

$$
\begin{aligned}
& g_{01} D_{0} D_{1}-g_{10} D_{1} D_{0}=x_{0} D_{1}-x_{1} D_{0} \\
& g_{12} D_{1} D_{2}-g_{21} D_{2} D_{1}=x_{1} D_{2}-x_{2} D_{1} \\
& g_{20} D_{2} D_{0}-g_{02} D_{0} D_{2}=x_{2} D_{0}-x_{0} D_{2}
\end{aligned}
$$

with boundary conditions on the states $\langle 0|$ and $|0\rangle$

$$
\langle 0|\left(x_{\nu}-L_{\nu}^{\mu} D_{\mu}\right)=0 \quad \text { and } \quad\left(x_{\nu}+R_{\nu}^{\mu} D_{\mu}\right)|0\rangle=0 .
$$

As shown in ref. [9], for $N=2$, one has Fock representations of the algebra for any bulk and boundary rates. The situation is going to be very different for $N=3$. The reason is that the number of boundary conditions determined by the matrices $L_{\mu}^{\nu}$ and $R_{\mu}^{\nu}$ is in general too large. For each of $L$ and $R$ one has to consider all the cases enumerated in the previous section (see eqs. (4.6-12)). We now make some transformations which exploit the fact that the $x_{\mu}$ 's are still free parameters.

Let us first consider words of length 1 :

$$
\left\langle 0\left|D_{\mu}\right| 0\right\rangle=\delta_{\mu} .
$$

From equation (5.3), it follows that

$$
\left(L_{\nu}^{\mu}+R_{\nu}^{\mu}\right) \delta_{\mu}=0
$$

and

$$
x_{\nu}=\frac{1}{2}\left(L_{\nu}^{\mu}-R_{\nu}^{\mu}\right) \delta_{\mu} .
$$

We have to keep in mind that

$$
B_{\mu}^{\nu}=L_{\mu}^{\nu}+R_{\mu}^{\nu}
$$

is an intensity matrix with cofactors $\mathcal{B}_{\mu}$. This implies (using eqs. (2.3-6)) that

$$
\delta_{\mu}=\xi \mathcal{B}_{\mu}
$$

and

$$
\begin{aligned}
x_{\nu} & =\frac{\xi}{2}\left(L_{\nu}^{\mu}-R_{\nu}^{\mu}\right) \mathcal{B}_{\mu} \\
& =\xi\left(L_{\nu}^{\mu} \mathcal{R}_{\mu}-R_{\nu}^{\mu} \mathcal{L}_{\mu}\right) .
\end{aligned}
$$


Here $\xi$ is an arbitrary parameter and $\mathcal{R}_{\mu}\left(\mathcal{L}_{\mu}\right)$ are the cofactors of the intensity matrix $R_{\nu}^{\mu}\left(L_{\nu}^{\mu}\right)$. The expressions for the various cofactors can be obtained using eq. (2.7). Since $R_{\nu}^{\mu}$ and $L_{\nu}^{\mu}$ are intensity matrices, it follows from eq.(5.3) that

$$
\sum_{\nu=0}^{2} x_{\nu}=0 .
$$

It is interesting to note that if we have a Fock representation, the ratio of the currents of particles 1 and 2 is already known. It is easy to show that

$$
J_{i}=x_{i} \frac{\left\langle 0\left|C^{L-1}\right| 0\right\rangle}{\left\langle 0\left|C^{L}\right| 0\right\rangle}, \quad i=1,2
$$

where

$$
C=\sum_{\mu=0}^{2} D_{\mu}
$$

This implies

$$
\frac{J_{1}}{J_{2}}=\frac{x_{1}}{x_{2}}
$$

where $x_{1} / x_{2}$ is given by the boundary conditions (see eq. (5.9)).

In deriving (5.11) we have used the following expression for the current density [5, 价

$$
\begin{aligned}
j_{1} & =g_{10} D_{1} D_{0}+g_{12} D_{1} D_{2}-g_{01} D_{0} D_{1}-g_{21} D_{2} D_{1} \\
& =-\left(x_{0}+x_{2}\right) D_{1}+x_{1}\left(D_{0}+D_{2}\right)=x_{1} C,
\end{aligned}
$$

and a similar equation for $j_{2}$. We also used the definition

$$
J_{i}=\left\langle j_{i}\right\rangle
$$

It is easy to find the one-dimensional representations of the algebra (5.2). Using eq. (5.4), we get the following conditions on the rates:

$$
\begin{aligned}
g_{01}-g_{10} & =y_{0}-y_{1} \\
g_{12}-g_{21} & =y_{1}-y_{2} \\
g_{20}-g_{02} & =y_{2}-y_{0}
\end{aligned}
$$

where

$$
y_{\mu}=\frac{x_{\mu}}{\delta_{\mu}}
$$


are given by the boundary conditions (see eqs. (5.8) and (5.9)). Notice that eqs. (5.16) give one relation between the bulk rates:

$$
g_{01}+g_{12}+g_{20}=g_{10}+g_{21}+g_{02}
$$

and two relations between boundary and bulk rates.

Before we start looking for the cases in which the Fock representations of the algebra (5.2) exist, it is useful to bring it into a different form. We denote

$$
u_{0}=\frac{y_{1}-y_{2}}{g_{21}}+1-q_{0}, \quad u_{1}=\frac{y_{2}-y_{0}}{g_{02}}+1-q_{1}, \quad u_{2}=\frac{y_{0}-y_{1}}{g_{10}}+1-q_{2}
$$

and also

$$
\begin{array}{ll}
v_{10}=1-q_{2}+\left(y_{0} / g_{10}\right) & v_{01}=1-q_{2}-\left(y_{1} / g_{10}\right) \\
v_{21}=1-q_{0}+\left(y_{1} / g_{21}\right) & v_{12}=1-q_{0}-\left(y_{2} / g_{21}\right) \\
v_{02}=1-q_{1}+\left(y_{2} / g_{02}\right) & v_{20}=1-q_{1}-\left(y_{0} / g_{02}\right)
\end{array} .
$$

The $q_{\mu}$ 's and $y_{\nu}$ 's in the above are defined in equations (4.22) and (5.17) respectively. We also define new generators $H_{\mu}$

$$
D_{\mu}=\delta_{\mu}\left(1+H_{\mu}\right)
$$

With these new definitions, the algebra relations and the action of the generators on the vacuum are

$$
\begin{aligned}
& q_{2} H_{0} H_{1}-H_{1} H_{0}=u_{2}+v_{10} H_{1}+v_{01} H_{0} \\
& q_{0} H_{1} H_{2}-H_{2} H_{1}=u_{0}+v_{21} H_{2}+v_{12} H_{1} \\
& q_{1} H_{2} H_{0}-H_{0} H_{2}=u_{1}+v_{02} H_{0}+v_{20} H_{2}
\end{aligned}
$$

with

$$
\begin{gathered}
\left\langle 0\left|H_{0}\right| 0\right\rangle=\left\langle 0\left|H_{1}\right| 0\right\rangle=\left\langle 0\left|H_{2}\right| 0\right\rangle=0 \\
\left(\mathcal{L}_{0} / \delta_{0}\right)\langle 0| H_{1}=\left(\mathcal{L}_{1} / \delta_{1}\right)\langle 0| H_{0} ; \quad\left(\mathcal{L}_{0} / \delta_{0}\right)\langle 0| H_{2}=\left(\mathcal{L}_{2} / \delta_{2}\right)\langle 0| H_{0} \\
\left(\mathcal{R}_{0} / \delta_{0}\right) H_{1}|0\rangle=\left(\mathcal{R}_{1} / \delta_{1}\right) H_{0}|0\rangle ; \quad\left(\mathcal{R}_{0} / \delta_{0}\right) H_{2}|0\rangle=\left(\mathcal{R}_{2} / \delta_{2}\right) H_{0}|0\rangle .
\end{gathered}
$$

The algebra (5.22) is of a special case of the ones defined in eq. (1.24) 12, 13]. For the latter one can show that For the latter one can show that if the coefficients are generic, then for $N \geq 3$ the algebra is finite dimensional. For example there are 28 independent monomials for $N=3$. Therefore one can already understand that the $N=3$ problem is different from the one for $N=2$. The next point to note is the role of boundary rates for $N=3$. They 
appear explicitly in eq. (5.24) but also through the $y_{\mu}$ 's in the coefficients of the algebra (5.22) (see eq. (5.20)).

In the next section we ask the question if the algebra (5.22) has representations in the generic case (when the principal cofactors of the boundary matrices are non-zero) aside from the one-dimensional one, which corresponds to $u_{\mu}=0$ and $H_{\mu}=0$. We shall show that one can have representations of dimension at most 2. In general, it is not possible to have higher dimensional representations because the number of constraints coming from the algebra relations is larger that the number of boundary and bulk rates. In the subsequent sections, we will make a systematic investigation of the cases where some or all of the principal minors vanish.

\section{Generic 3-state exclusion model: boundary in- tensity matrices have non-zero minors}

In this section we consider the question when the algebra (5.22) has Fock representations defined by eqs $(5.23-4)$ under the assumption that the $\mathcal{R}_{\mu}$ and $\mathcal{L}_{\mu}$ are non-zero. This is a very technical section and the reader not interested in the method of answering the question can proceed directly to the end of the section where the result is given. For our specific purpose it is convenient to rewrite the algebra in a slightly different way using new notation.

Let

$$
q_{\nu}=Q_{\nu}^{2} ; \quad \lambda_{i}=\frac{\mathcal{L}_{i}}{\mathcal{L}_{0}} \frac{\delta_{0}}{\delta_{i}}, \quad \mu_{i}=\frac{\mathcal{R}_{i}}{\mathcal{R}_{0}} \frac{\delta_{0}}{\delta_{i}}, \quad \alpha_{i}^{2}=\frac{\lambda_{i}}{\mu_{i}}, \quad(\nu=0,1,2 ; i=1,2)
$$

and instead of the generators $H_{\nu}$ use $L_{\nu}$ defined as follows:

$$
D_{0}=\delta_{0}\left(1+L_{0}\right), \quad D_{i}=\delta_{1}\left(1+\lambda_{1} L_{1}\right), \quad D_{2}=\delta_{2}\left(1+\mu_{2} L_{2}\right) .
$$

The algebra relations are

$$
\begin{aligned}
& Q_{2}^{2} L_{0} L_{1}-L_{1} L_{0}=m_{2}+n_{10} L_{1}+n_{01} L_{0} \\
& Q_{0}^{2} L_{1} L_{2}-L_{2} L_{1}=m_{0}+n_{21} L_{2}+n_{12} L_{1} \\
& Q_{1}^{2} L_{2} L_{0}-L_{0} L_{2}=m_{1}+n_{02} L_{0}+n_{20} L_{2}
\end{aligned}
$$


and the conditions for a Fock representation are

$$
\begin{aligned}
& \langle 0| L_{1}=\langle 0| L_{0}, \quad\langle 0| L_{2} \quad=\quad \alpha_{1}^{2}\langle 0| L_{0}, \\
& L_{2}|0\rangle=L_{0}|0\rangle, \quad L_{1}|0\rangle=\alpha_{2}^{2} L_{0}|0\rangle, \\
& \left\langle 0\left|L_{\nu}\right| 0\right\rangle=0, \quad(\nu=0,1,2)
\end{aligned}
$$

This algebra depends on 14 parameters, and after setting the time scale, we are effectively left with 13 . For the physical problem, the $m_{\nu}$ 's and $n_{\mu \nu}$ 's are related to the rates:

$$
\begin{aligned}
& m_{0}=\frac{1}{\lambda_{1} \mu_{2}}\left(\frac{y_{1}-y_{2}}{g_{12}}+1-Q_{0}^{2}\right) \\
& m_{1}=\frac{1}{\mu_{2}}\left(\frac{y_{2}-y_{0}}{g_{02}}+1-Q_{1}^{2}\right) \\
& m_{2}=\frac{1}{\lambda_{1}}\left(\frac{y_{0}-y_{1}}{g_{10}}+1-Q_{2}^{2}\right)
\end{aligned}
$$

and

$$
\begin{aligned}
n_{10}=1-Q_{2}^{2}+\frac{y_{0}}{g_{10}}, & n_{01}=\frac{1}{\lambda_{1}}\left(1-Q_{2}^{2}-\frac{y_{1}}{g_{10}}\right), \\
n_{02}=\frac{1}{\mu_{2}}\left(1-Q_{1}^{2}+\frac{y_{2}}{g_{02}}\right), & n_{20}=\left(1-Q_{1}^{2}-\frac{y_{0}}{g_{02}}\right), \\
n_{21}=\frac{1}{\lambda_{1}}\left(1-Q_{0}^{2}+\frac{y_{1}}{g_{21}}\right), & n_{12}=\frac{1}{\mu_{2}}\left(1-Q_{0}^{2}-\frac{y_{2}}{g_{21}}\right) .
\end{aligned}
$$

At this point two strategies are possible. One can look for vacuum expectation values of words of different lengths and find consistency conditions for the 14 paramenters; or, one can look for matrix representations of the algebra and in this way obtain consistency conditions among the parameters. We shall do both - we shall take matrix representations and indicate which of the consistency conditions on the matrix elements also come from specific vacuum expectation values of words of given length. In order to do so, it is convenient to work with paramaters $p_{\mu \nu}$ and $r_{\mu}$ introduced below instead of the $m_{\mu}$ and $n_{\mu \nu}$ introduced earlier.

$$
\begin{aligned}
m_{0} & =Q_{0} \alpha_{1} \alpha_{2} N\left(Q_{0} /\left(\alpha_{1} \alpha_{2}\right)\right) r_{0} \\
m_{1} & =Q_{1} \alpha_{1} N\left(Q_{1} \alpha_{1}\right) r_{1} \\
m_{2} & =Q_{2} \alpha_{2} N\left(Q_{2} \alpha_{2}\right) r_{2} \\
n_{01} & =Q_{2} \alpha_{2} N\left(Q_{2}^{2} \alpha_{2}\right) p_{01} \\
n_{10} & =Q_{2} N\left(Q_{2}^{2} \alpha_{2}\right) p_{10} \\
n_{20} & =Q_{1} N\left(Q_{1}^{2} \alpha_{1}\right) p_{20} \\
n_{02} & =Q_{1} \alpha_{1} N\left(Q_{1}^{2} \alpha_{1}\right) p_{02} \\
n_{21} & =Q_{0} \alpha_{2} N\left(Q_{0}^{2} /\left(\alpha_{1} \alpha_{2}\right)\right) p_{21} \\
n_{12} & =Q_{0} \alpha_{1} N\left(Q_{0}^{2} /\left(\alpha_{1} \alpha_{2}\right)\right) p_{12}
\end{aligned}
$$


The constraints on the parameters we shall obtain in what follows look much simpler in terms of the new parameters. In eqs. (6.7) and in the what follows we use the notation

$$
M(x)=x+\frac{1}{x} \quad \text { and } \quad N(x)=x-\frac{1}{x} .
$$

We consider a three-dimensional representation of the algebra eq. (6.3). We make this choice since we are going to prove that in the the generic case, the two-dimensional representation is the largest we can have. Using the boundary conditions (6.4) we can bring the matrix representation of the generators $L_{\mu}$ to a tridiagonal form by means of a similarity transformation by an orthogonal matrix, just as it was done for the $N=2$ case in [9]. One can show that with

$$
\langle 0| \doteq(1,0,0) \quad \text { and } \quad|0\rangle \doteq(1,0,0)^{T}
$$

(where $T$ denotes transposition) the generators then take on the expressions

$$
\begin{gathered}
L_{0} \doteq\left(\begin{array}{ccc}
0 & \sqrt{f_{1}} & 0 \\
\sqrt{f_{1}} & c_{1}^{(0)} & \sqrt{f_{2}} \\
0 & \sqrt{f_{2}} & c_{2}^{(0)}
\end{array}\right) \\
L_{1} \doteq\left(\begin{array}{ccc}
0 & \sqrt{f_{1}} & 0 \\
\alpha_{2}^{2} \sqrt{f_{1}} & \alpha_{2} c_{1}^{(1)} & \frac{\sqrt{f_{2}}}{Q_{2}^{2}} \\
0 & \alpha_{2}^{2} Q_{2}^{2} \sqrt{f_{2}} & \alpha_{2} c_{2}^{(1)}
\end{array}\right) \\
L_{2} \doteq\left(\begin{array}{ccc}
0 & \alpha_{1}^{2} \sqrt{f_{1}} & 0 \\
\sqrt{f_{1}} & \alpha_{1} c_{1}^{(2)} & \alpha_{1}^{2} Q_{1}^{2} \sqrt{f_{2}} \\
0 & \frac{\sqrt{f_{2}}}{Q_{1}^{2}} & \alpha_{1} c_{2}^{(2)}
\end{array}\right)
\end{gathered}
$$

Notice that a one-dimensional representation is obtained if $f_{1}=0$, a two-dimensional one if $f_{2}=0, f_{1} \neq 0$ and a three-dimensional one if $f_{1} \neq$ $0, f_{2} \neq 0 . f_{i}$ and $c_{i}^{(j)}$ are still to be determined from the algebra relations, the Fock conditions (6.4) having already been taken into account.

We now insert the $3 \times 3$ matrices (6.9) in the algebraic relations (6.3) and from the equality of each matrix element $(i, j)$ on the left- and righthand sides, get the following equations. From the $(1,1)$ matrix element, we obtain

$$
r_{0}=r_{1}=r_{2}=f_{1} .
$$


From the matrix elements $(1,2),(2,1)$ and $(2,2)$ we get

$$
\begin{aligned}
& c_{1}^{(0)}=M\left(\alpha_{2} Q_{2}\right) p_{10}+M\left(Q_{2}\right) p_{01}=M\left(Q_{1}\right) p_{02}+M\left(\alpha_{1} Q_{1}\right) p_{20} \\
& c_{1}^{(1)}=M\left(Q_{2}\right) p_{10}+M\left(\alpha_{2} Q_{2}\right) p_{01}=M\left(Q_{0} /\left(\alpha_{1} \alpha_{2}\right)\right) p_{21}+M\left(Q_{0}\right) p_{12} \\
& c_{1}^{(2)}=M\left(\alpha_{1} Q_{1}\right) p_{02}+M\left(Q_{1}\right) p_{20}=M\left(Q_{0} /\left(\alpha_{1} \alpha_{2}\right)\right) p_{12}+M\left(Q_{0}\right) p_{21} \\
& \frac{N\left(Q_{2}^{3} \alpha_{2}\right)}{N\left(\alpha_{2}\right) M\left(Q_{2}\right)} f_{2}=f_{1}+p_{10}^{2}+p_{01}^{2}+M\left(Q_{2}^{2} \alpha_{2}\right) p_{01} p_{10} \\
& \frac{N\left(\left(\alpha_{1} \alpha_{2}\right) / Q_{0}^{3}\right)}{N\left(\alpha_{1} \alpha_{2}\right) M\left(Q_{0}\right)} f_{2}=f_{1}+p_{12}^{2}+p_{21}^{2}+M\left(\left(\alpha_{1} \alpha_{2}\right) / Q_{0}^{2}\right) p_{21} p_{12} \\
& \frac{N\left(Q_{1}^{3} \alpha_{1}\right)}{N\left(\alpha_{1}\right) M\left(Q_{1}\right)} f_{2}=f_{1}+p_{20}^{2}+p_{02}^{2}+M\left(Q_{1}^{2} \alpha_{1}\right) p_{02} p_{20} .
\end{aligned}
$$

Let us pause here for a moment. Equations (6.10) and (6.11) can also be obtained from the vacuum expectation values of words of length two and three. The 1-dimensional representation is obtained from the condition $f_{1}=0$ and the 2-dimensional representation is obtained by setting $f_{2}=0$ in (6.12). From eqs. (6.11) and (6.12) the $p_{\mu \nu}$ are completely determined as are the $r_{\mu}$ (eq. (6.10)). This means that in the algebra (6.3), the free parameters are $\alpha_{1}, \alpha_{2}$ and $Q_{\mu}, \mu=0,1,2$. The next logical step would be to solve the equations and look for solutions with positive rates. This is a difficult exercise. For a given physical problem, where some conditions on the rates are given, the problem is simpler since there are fewer parameters.

We now go on with the rest of the matrix elements $(1,3),(2,3),(3,1)$, $(3,2)$ and $(3,3)$. We get:

$$
\begin{aligned}
Q_{0}^{2} Q_{1}^{2} Q_{2}^{2}=1, \\
c_{2}^{(0)}=\frac{M\left(Q_{1}\right)}{N\left(\alpha_{1} Q_{1}^{4}\right)}\left(\left(N\left(\alpha_{1}^{2} Q_{1}^{4}\right)-N\left(Q_{1}^{2}\right)\right) p_{20}+M\left(Q_{1}\right) N\left(Q_{1} \alpha_{1}\right) p_{02}\right) \\
=\frac{M\left(Q_{2}\right)}{N\left(\alpha_{2} Q_{2}^{4}\right)}\left(\left(N\left(\alpha_{2}^{2} Q_{2}^{4}\right)-N\left(Q_{2}^{2}\right)\right) p_{10}+M\left(Q_{2}\right) N\left(Q_{2} \alpha_{2}\right) p_{01}\right) \\
c_{2}^{(1)}=\frac{M\left(Q_{2}\right)}{N\left(\alpha_{2} Q_{2}^{4}\right)}\left(\left(N\left(\alpha_{2}^{2} Q_{2}^{4}\right)-N\left(Q_{2}^{2}\right)\right) p_{01}+M\left(Q_{2}\right) N\left(Q_{2} \alpha_{2}\right) p_{10}\right) \\
=\frac{M\left(Q_{0}\right)}{N\left(Q_{0}^{4} /\left(\alpha_{1} \alpha_{2}\right)\right)}\left(\left(N\left(\frac{Q_{0}^{4}}{\alpha_{1} \alpha_{2}}\right)-N\left(Q_{0}^{2}\right)\right) p_{21}+M\left(Q_{0}\right) N\left(\frac{Q_{0}}{\alpha_{1} \alpha_{2}}\right) p_{12}\right) \\
c_{2}^{(2)}=\frac{M\left(Q_{1}\right)}{N\left(\alpha_{1} Q_{1}^{4}\right)}\left(\left(N\left(\alpha_{1}^{2} Q_{1}^{4}\right)-N\left(Q_{1}^{2}\right)\right) p_{02}+M\left(Q_{1}\right) N\left(Q_{2} \alpha_{2}\right) p_{20}\right) \\
=\frac{M\left(Q_{0}\right)}{N\left(\frac{Q_{0}^{4}}{\alpha_{1} \alpha_{2}}\right)}\left(\left(N\left(\frac{Q_{0}^{4}}{\alpha_{1} \alpha_{2}}\right)-N\left(Q_{0}^{2}\right)\right) p_{12}+M\left(Q_{0}\right) N\left(\frac{Q_{0}}{\alpha_{1} \alpha_{2}}\right) p_{21}\right)
\end{aligned}
$$


and

$$
\begin{aligned}
f_{2} & =-f_{1}+\frac{1}{N\left(\alpha_{2} Q_{2}\right)}\left(N\left(Q_{2}\right) c_{0}^{(2)} c_{1}^{(2)}-N\left(\alpha_{2} Q_{2}^{2}\right)\left(p_{01} c_{0}^{(2)}+p_{10} c_{1}^{(2)}\right)\right) \\
& =-f_{1}+\frac{1}{N\left(\frac{Q_{0}}{\alpha_{1} \alpha_{2}}\right)}\left(N\left(Q_{0}\right) c_{1}^{(2)} c_{2}^{(2)}-N\left(Q_{0}^{2} /\left(\alpha_{1} \alpha_{2}\right)\right)\left(p_{12} c_{1}^{(2)}+p_{21} c_{2}^{(2)}\right)\right) \\
& =-f_{1}+\frac{1}{N\left(\alpha_{1} Q_{1}\right)}\left(N\left(Q_{1}\right) c_{0}^{(2)} c_{2}^{(2)}-N\left(\alpha_{1} Q_{1}^{2}\right)\left(p_{20} c_{2}^{(2)}+p_{02} c_{0}^{(2)}\right)\right)
\end{aligned}
$$

Equations (6.11) and (6.14) give a system of six homogeneous equations for the $p_{\mu \nu}$ with determinant generically different from zero. In order to have a non-trivial solution for the $p_{\mu \nu}$ 's, we must set this determinant to zero, thus introducing an extra condition on the rates. This leaves one of them (say $\left.p_{10}\right)$ free. The same equations determine $c_{i}^{(\mu)}(i=1,2, \mu=0,1,2)$. We are thus left with eight equations: three from (6.12), one from (6.13), three from (6.15), and one determinantal condition for the seven unknowns $f_{2}, p_{10}, Q_{\mu}$ and $\alpha_{i}$. (We can take $f_{1}=1$ in eq. (6.10) to set the time scale.) This implies that we cannot, generically have a 3 -dimensional representation. This also implies that the algebra (6.3) with the Fock conditions (6.4) can have representations of dimensions 1 and 2 only. The conditions on the parameters are not neat but this was so even for the case $N=2$ [9].

We now come back to the physical problem. The algebra (5.22) contains 9 parameters $-q_{0}, q_{1}, q_{2}$ and the ratios $\left(y_{0} / g_{10}\right),\left(y_{0} / g_{02}\right),\left(y_{1} / g_{10}\right),\left(y_{1} / g_{21}\right)$, $\left(y_{2} / g_{02}\right)$ and $\left(y_{2} / g_{21}\right)$; the boundary conditions (5.24) depend on 4 others, the $\lambda_{i}$ 's and $\mu_{i}$ 's for $i=1,2$. This makes a total of 13 parameters. Therefore for the physical problem, the case of 2-dimensional representations stays interesting since the algebraic varieties on which the solution set exists is not trivial.

\section{The two non-zero cofactors case}

In the last section we considered the generic case where all the cofactors $\mathcal{L}_{\mu}$ and $\mathcal{R}_{\mu}$ are non-zero and we found that the algebra (5.22) had Fock representations defined by eqs. (5.23) and (5.24) of dimension 1 and 2 only. We should have continued our study and looked at all cases when some or all of the cofactors vanish. In this section we shall confine ourselves only to the case

$$
\mathcal{L}_{0}=\mathcal{L}_{2}=\mathcal{R}_{0}=\mathcal{R}_{1}=0, \quad \mathcal{L}_{1} \neq 0, \mathcal{R}_{2} \neq 0
$$


We had in mind the physical application of ref. [5]. The interested reader can easily duplicate the calculations presented in this section for the other cases. With (7.1), the Fock representations have to satisfy the following simple simple conditions

$$
\left\langle 0\left|H_{0}=\left\langle 0\left|H_{2}=0 ; \quad H_{0}\right| 0\right\rangle=H_{1}\right| 0\right\rangle=0, \quad\left\langle 0\left|H_{\mu}\right| 0\right\rangle=0 .
$$

In order to find out in which cases the algebra (5.22) has Fock representations defined by eq. (7.2), we take vacuum expectation values for monomials of different degree.

Monomials of degree 2 ( consider the algebra relations between $\langle 0|$ and $|0\rangle$ ) give:

$$
u_{1}=u_{2}=0 .
$$

Monomials of degree 3 (consider $\left\langle 0\left|H_{1} H_{0} H_{2}\right| 0\right\rangle$ and commute $H_{0}$ through to the left and the right) give

$$
v_{10}=v_{20}=v .
$$

Monomials of degree $4,\left\langle 0\left|H_{1} H_{1} H_{0} H_{2}\right| 0\right\rangle$ and $\left\langle 0\left|H_{1} H_{2} H_{0} H_{2}\right| 0\right\rangle$ give

$$
q_{0} v_{01}=q_{2} v_{21} \quad \text { and } \quad q_{0} v_{02}=q_{1} v_{12}
$$

respectively. We have to distinguish several cases

- a) $v_{12}$ and $v_{21}$ are non-zero.

Monomials of degree 5 (consider $\left\langle 0\left|H_{1} H_{0} H_{1} H_{2} H_{2}\right| 0\right\rangle$ ) give

$$
q_{1}=q_{2}=q .
$$

Taking into account relations (7.3-6) the algebra (5.22) becomes

$$
\begin{aligned}
q H_{0} H_{1}-H_{1} H_{0} & =v H_{1}+\frac{q}{q_{0}} v_{21} H_{0} \\
q H_{2} H_{0}-H_{0} H_{2} & =\frac{q}{q_{0}} v_{12} H_{0}+v H_{2} \\
q_{0} H_{1} H_{2}-H_{2} H_{1} & =u_{0}+v_{12} H_{1}+v_{21} H_{2}
\end{aligned}
$$

It is useful to change variables

$$
H_{0}=-v \mathcal{N}, \quad H_{1}=\frac{u_{0}}{v_{12}} \mathcal{A}, \quad H_{2}=\frac{u_{0}}{v_{21}} \mathcal{B}, \quad z_{2}=\frac{u_{0} q_{0}}{v_{12} v_{21}}
$$


in terms of which, the algebra becomes

$$
\begin{aligned}
& \text { a) } z_{2}\left(\mathcal{A B}-\frac{1}{q_{0}} \mathcal{B} \mathcal{A}\right)=1+\mathcal{A}+\mathcal{B} \\
& \text { b) } \mathcal{A N}-q \mathcal{N} \mathcal{A}=\mathcal{A}-\frac{q}{z_{2}} \mathcal{N} \\
& \text { c) } \quad \mathcal{N B}-q \mathcal{B N}=\mathcal{B}-\frac{q^{2}}{z_{2}} \mathcal{N} \text {. }
\end{aligned}
$$

This algebra has the representations

$$
\langle 0|\mathcal{N}=0=\mathcal{N}| 0\rangle, \quad\langle 0|\mathcal{B}=0=\mathcal{A}| 0\rangle .
$$

We can now use the results of ref. [9] to find the following representation of (7.9a):

$$
\mathcal{A} \doteq\left(\begin{array}{cccc}
a_{1} & f_{1} & 0 & 0 \ldots \\
0 & a_{2} & f_{2} & 0 \ldots \\
0 & 0 & a_{3} & f_{3} \ldots \\
0 & 0 & 0 & a_{4} \ldots \\
\vdots & \vdots & \vdots & \ddots
\end{array}\right), \quad \mathcal{B}=\mathcal{A}^{T} \doteq\left(\begin{array}{cccc}
a_{1} & 0 & 0 & 0 \ldots \\
f_{1} & a_{2} & 0 & 0 \ldots \\
0 & f_{2} & a_{3} & 0 \ldots \\
0 & 0 & f_{3} & a_{4} \ldots \\
\vdots & \vdots & \vdots & \ddots
\end{array}\right)
$$

where

$$
\begin{array}{cc}
a_{n}=z_{2}^{-1}\{n-1\}_{\lambda}, & f_{n}^{2}=z_{2}^{-2}\{n\}_{\lambda}\left(z_{2}+\{n-1\}_{\lambda}\right), \\
\{n\}_{\lambda}=\frac{1-\lambda^{n}}{1-\lambda}, n \geq 1,\{0\}_{\lambda}=1 & \lambda=q_{0}^{-1} .
\end{array}
$$

We have introduced the symbol $z_{2}$ in keeping with the notation of ref. [9]. $\mathcal{A}^{T}$ is the transpose of matrix $\mathcal{A}$. There are two cases when we can have representations for $\mathcal{N}$ in eqs. (7.9):

$$
\text { a.1) } \quad q=q_{0}, \quad v_{01}=v_{21}, \quad v_{02}=v_{12}
$$

when

$$
\mathcal{N} \doteq\left(\begin{array}{cccc}
h_{1} & 0 & 0 & \ldots \\
0 & h_{2} & 0 & \ldots \\
0 & 0 & h_{3} & \ldots \\
\vdots & \vdots & \vdots & \ddots
\end{array}\right)
$$

with

$$
h_{n}=\{n-1\}_{q}
$$


and

$$
\text { a.2) } \quad q=0, \quad q_{0} \neq 0, \quad v_{01}=v_{02}=0
$$

with

$$
h_{n}=\{n-1\}_{q \rightarrow 0}=1-\delta_{n 1}
$$

- b) We consider the case (see eq. (7.5))

$$
v_{01}=v_{02}=v_{12}=v_{21}=0, \quad v_{10}=v_{20}=v, \quad u_{1}=u_{2}=0 .
$$

Monomials of degree 5 give (see eq. (7.6))

$$
q_{1}=q_{2}=q .
$$

With (7.18) and (7.19) the algebra (5.22) becomes

$$
\begin{aligned}
q H_{0} H_{1}-H_{1} H_{0} & =v H_{1} \\
q H_{2} H_{0}-H_{0} H_{2} & =v H_{2} \\
q_{0} H_{1} H_{2}-H_{2} H_{1} & =u_{0} .
\end{aligned}
$$

We make the transformation

$$
H_{0}=-v \mathcal{N}, \quad H_{1}=\sqrt{\frac{u_{0}}{q_{0}}} \mathcal{A}, \quad H_{2}=\sqrt{\frac{u_{0}}{q_{0}}} \mathcal{B}, \quad \lambda=\frac{1}{q_{0}}
$$

and get the algebra relations

$$
\begin{aligned}
\mathcal{A B}-\lambda \mathcal{B} \mathcal{A} & =1 \\
\mathcal{A N}-q \mathcal{N} \mathcal{A} & =\mathcal{A} \\
\mathcal{N B}-q \mathcal{B N} & =\mathcal{B}
\end{aligned}
$$

and

$$
\mathcal{A}|0\rangle=\mathcal{N}|0\rangle=0=\langle 0| \mathcal{B}=\langle 0| \mathcal{N} .
$$

The Fock representation of this algebra (matrices satisfying (7.22) and (7.23)) is:

$$
\mathcal{A}=\mathcal{B}^{T} \doteq\left(\begin{array}{cccc}
0 & g_{1} & 0 & \ldots \\
0 & 0 & g_{2} & \\
0 & 0 & 0 & \\
\vdots & & & \ddots
\end{array}\right), \quad \mathcal{N} \doteq\left(\begin{array}{cccc}
h_{1} & 0 & 0 & \ldots \\
0 & h_{2} & 0 & \\
0 & 0 & h_{3} & \\
\vdots & & & \ddots
\end{array}\right)
$$

where

$$
g_{n}^{2}=\{n\}_{\lambda}, \quad h_{n}=\{n-1\}_{q} .
$$


- c) We consider the case (see (eq.(7.5))

$$
v_{21}=v_{01}=0 .
$$

The algebra (5.22) again with $q_{1}=q_{2}=q$ becomes

$$
\begin{aligned}
q H_{0} H_{1}-H_{1} H_{0} & =v H_{1} \\
q H_{2} H_{0}-H_{0} H_{2} & =\frac{q}{q_{0}} v_{12} H_{0}+v H_{2} \\
q_{0} H_{1} H_{2}-H_{2} H_{1} & =u_{0}+v_{12} H_{1}
\end{aligned}
$$

We make the substitutions

$$
H_{0}=-v \mathcal{N}, \quad H_{1}=\frac{u_{0}}{v_{12}} \mathcal{A}, \quad H_{2}=\frac{v_{12}}{q_{0}} \mathcal{B}, \quad \lambda=\frac{1}{q_{0}}
$$

to get the algebra

$$
\begin{array}{rlc}
\mathcal{A B}-\lambda \mathcal{B} \mathcal{A} & = & 1+\mathcal{A} \\
\mathcal{A N}-q \mathcal{N} \mathcal{A} & = & \mathcal{A} \\
\mathcal{N B}-q \mathcal{B N} & = & \mathcal{B}-q \mathcal{N} .
\end{array}
$$

This algebra has the representations

$$
\mathcal{A} \doteq\left(\begin{array}{cccc}
0 & f_{1} & 0 & \ldots \\
0 & 0 & f_{2} & \\
0 & 0 & 0 & \\
\vdots & & & \ddots
\end{array}\right), \quad \mathcal{B} \doteq\left(\begin{array}{cccc}
b_{1} & 0 & 0 & \ldots \\
f_{1} & b_{2} & 0 & \\
0 & f_{2} & b_{3} & \\
\vdots & & & \ddots
\end{array}\right)
$$

where

$$
f_{n}^{2}=\{n\}_{\lambda}, \quad b_{n}=\{n-1\}_{\lambda}
$$

and if

$$
\text { c.1) } \quad q=q_{0}=\lambda^{-1} \text {, }
$$

then

$$
\mathcal{N} \doteq\left(\begin{array}{cccc}
h_{1} & 0 & 0 & \cdots \\
0 & h_{2} & 0 & \\
0 & 0 & h_{3} & \\
\vdots & & & \ddots
\end{array}\right)
$$

where

$$
h_{k}=\{k-1\}_{\lambda} .
$$

If

$$
\text { c.2) } \quad q=0 \text {, }
$$


which implies $v_{02}=0$ one has to take

$$
h_{k}=\{k-1\}_{\lambda \rightarrow 0}=1-\delta_{k 1} .
$$

In order to help the reader, we shall summarize the results. The algebra (5.22) has Fock representations defined by

$$
\left\langle 0\left|H_{2}=\left\langle 0\left|H_{0}=0=H_{0}\right| 0\right\rangle=H_{1}\right| 0\right\rangle
$$

if (necessary condition)

$$
u_{1}=u_{2}=0, \quad v_{10}=v_{20}=v, \quad q_{1}=q_{2}=q,
$$

in which case the algebra relations become

$$
\begin{aligned}
q H_{0} H_{1}-H_{1} H_{0} & =v H_{1}+v_{01} H_{0} \\
q H_{2} H_{0}-H_{0} H_{2} & =v_{02} H_{0}+v H_{2} \\
q_{0} H_{1} H_{2}-H_{2} H_{1} & =u_{0}+v_{12} H_{1}+v_{21} H_{2}
\end{aligned}
$$

This algebra has Fock representations in five cases

1.

$$
v_{01}=v_{21}, \quad v_{02}=v_{12}, \quad q_{0}=q .
$$

With the notations

$$
H_{0}=-v \mathcal{N}, \quad H_{1}=\frac{u_{0}}{v_{12}} \mathcal{A}, \quad H_{2}=\frac{u_{0}}{v_{21}} \mathcal{B}, \quad z_{2}=\frac{u_{0} q_{0}}{v_{12} v_{21}},
$$

$\mathcal{A}$ and $\mathcal{B}$ are given by eq. (7.11-12) with $\lambda=q_{0}^{-1}$ and $\mathcal{N}$ by eqs. (7.14) with (7.15).

2 .

$$
v_{01}=v_{02}=q=0 .
$$

Same as case 1) except that $\mathcal{N}$ is given by eq. (7.14) with (7.17).

3.

$$
\begin{gathered}
v_{01}=v_{02}=v_{12}=v_{21}=0 . \\
H_{0}=-v \mathcal{N}, \quad H_{1}=\sqrt{\frac{u_{0}}{q_{0}}} \mathcal{A}, \quad H_{2}=\sqrt{\frac{u_{0}}{q_{0}}} \mathcal{B}, \quad \lambda=\frac{1}{q_{0}} .
\end{gathered}
$$

$\mathcal{A}, \mathcal{B}$ and $\mathcal{N}$ are given by eq. (7.24-5). 
4.

$$
\begin{gathered}
v_{01}=v_{21}=0, \quad q=q_{0}=\frac{1}{\lambda} . \\
H_{0}=-v \mathcal{N}, \quad H_{1}=\frac{u_{0}}{v_{12}} \mathcal{A}, \quad H_{2}=\frac{v_{12}}{q_{0}} \mathcal{B}, \quad \lambda=\frac{1}{q_{0}}
\end{gathered}
$$

$\mathcal{A}, \mathcal{B}$ and $\mathcal{N}$ are given by eq. (7.29) and $\mathcal{N}$ by eq. (7.31) with (7.32).

5.

$$
v_{02}=v_{01}=v_{21}=0, \quad q=0 .
$$

Everything as in case 4 ) except $\mathcal{N}$ is given by eq. (7.31) with eq.(7.33).

This closes the problem of Fock representations of the algebra (7.7). We now turn to the physical problem. This implies using the definitions (5.19) and (5.20) of the parameters $u_{\mu}$ and $v_{\mu \nu}$. We first have to give the boundary rates which give eq. (7.1). Using (2.7) we have the solution

$$
\begin{aligned}
L_{2}^{1} & =L_{0}^{1}=R_{1}^{2}=R_{0}^{2}=0 \\
\mathcal{L}_{1} & =L_{1}^{0} L_{0}^{2}+L_{1}^{2} L_{1}^{0}+L_{1}^{2} L_{2}^{0} \\
\mathcal{R}_{2} & =R_{0}^{1} R_{2}^{0}+R_{2}^{1} R_{1}^{0}+R_{2}^{1} R_{2}^{0}
\end{aligned}
$$

We now compute $\mathcal{B}_{\mu}$ and $x_{\mu}$ using eqs. (5.8) and (5.9):

$$
\begin{aligned}
& \delta_{0}=\xi\left(R_{0}^{1} L_{1}^{2}+L_{0}^{2} R_{0}^{1}+L_{0}^{2} R_{2}^{1}\right) \\
& \delta_{1}=\xi\left(R_{1}^{0} L_{0}^{2}+R_{1}^{0} L_{1}^{2}+R_{2}^{0} L_{1}^{2}+\mathcal{L}_{1}\right) \\
& \delta_{2}=\xi\left(R_{0}^{1} L_{2}^{0}+R_{2}^{1} L_{2}^{0}+R_{2}^{1} L_{1}^{0}+\mathcal{R}_{2}\right)
\end{aligned}
$$

and

$$
\begin{aligned}
& x_{0}=\xi\left(L_{0}^{2} \mathcal{R}_{2}-R_{0}^{1} \mathcal{L}_{1}\right) \\
& x_{1}=\xi\left(L_{1}^{2} \mathcal{R}_{2}+\left(R_{0}^{1}+R_{2}^{1}\right) \mathcal{L}_{1}\right) \\
& x_{2}=-\xi\left(\left(L_{0}^{2}+L_{1}^{2}\right) \mathcal{R}_{2}+R_{2}^{1} \mathcal{L}_{1}\right) .
\end{aligned}
$$

We now implement the conditions (7.35) to get

$$
y_{0}=0, \quad y_{1}=g_{10}(1-q), \quad y_{2}=-g_{02}(1-q) .
$$

We have to consider the 5 cases separately.

1. From (7.37) and (5.20) we get

$$
y_{1}=0=y_{2} .
$$

Taking into account the positivity of the rates, the definition (5.17) and the relation (5.6) this can be excluded. 
2. From eq. (7.38) and (5.19) we have

$$
g_{20}=0=g_{01}, \quad y_{0}=0, \quad y_{1}=g_{10}, \quad y_{2}=-g_{02} .
$$

Using the definitions (5.19) and (5.20) we get

$$
v_{10}=v_{20}=1
$$

and

$$
v_{12}=1-q_{0}+\frac{g_{02}}{g_{21}}, v_{21}=1-q_{0}+\frac{g_{10}}{g_{21}}, u_{0}=1-q_{0}+\frac{g_{10}+g_{02}}{g_{21}},
$$

and the following constraints on the rates coming from the definition (5.17) of $y_{\mu}$ and from from eq. (7.47):

$$
\begin{aligned}
L_{0}^{2} \mathcal{R}_{2} & =R_{0}^{1} \mathcal{L}_{1} \\
g_{10} & =\frac{L_{1}^{2} \mathcal{R}_{2}+\left(R_{0}^{1}+R_{2}^{1}\right) \mathcal{L}_{1}}{R_{1}^{0} L_{0}^{2}+R_{1}^{0} L_{1}^{2}+R_{2}^{0} L_{1}^{2}+\mathcal{L}_{1}} \\
g_{02} & =\frac{\left(L_{0}^{2}+L_{1}^{2}\right) \mathcal{R}_{2}+R_{2}^{1} \mathcal{L}_{1}}{R_{0}^{1} L_{2}^{0}+R_{2}^{1} L_{2}^{0}+R_{2}^{1} L_{1}^{0}+\mathcal{R}_{2}}
\end{aligned}
$$

3. As a consequence of eqs.(7.39) and (7.45) we find:

$$
\begin{gathered}
g_{10}=g_{02}, \quad g_{01}=g_{20}, \quad g_{12}-g_{21}=g_{10}-g_{01}, \\
y_{0}=0, \quad y_{1}=-y_{2}=g_{10}-g_{01}
\end{gathered}
$$

and

$$
v_{12}=0=v_{21}, \quad u_{0}=q_{0}-1, \quad v_{10}=v_{20}=1-q .
$$

From eq. (7.50) we find the following constraints on the rates:

$$
\begin{aligned}
L_{0}^{2} \mathcal{R}_{2} & =R_{0}^{1} \mathcal{L}_{1} \\
\frac{L_{1}^{2} \mathcal{R}_{2}+\left(R_{0}^{1}+R_{2}^{1}\right) \mathcal{L}_{1}}{R_{1}^{0} L_{0}^{2}+R_{1}^{0} L_{1}^{2}+R_{2}^{0} L_{1}^{2}+\mathcal{L}_{1}} & =\frac{\left(L_{0}^{2}+L_{1}^{2}\right) \mathcal{R}_{2}+R_{2}^{1} \mathcal{L}_{1}}{R_{0}^{1} L_{2}^{0}+R_{2}^{1} L_{2}^{0}+R_{2}^{1} L_{1}^{0}+\mathcal{R}_{2}} \\
& =g_{10}-g_{01} .
\end{aligned}
$$

4. No solutions if we want to maintain positivity of the rates. 
5. We find

$$
\begin{aligned}
& g_{21}+g_{10}=g_{12} \quad g_{20}=0=g_{01}, \\
& y_{0}=0, \quad y_{1}=g_{10}, \quad y_{2}=-g_{02}
\end{aligned}
$$

and

$$
v_{10}=v_{20}=1, \quad v_{12}=\frac{g_{02}-g_{10}}{g_{21}}, \quad u_{0}=\frac{g_{02}}{g_{21}} .
$$

The conditions on the rates can be derived from eqs. (7.54) and (5.17). Notice that this case is a specialization of case 3 . The representation is different because $z_{2}$ defined in eq. (7.8) and used for case 3 diverges $\left(v_{21}=0\right)$.

In Appendix A we consider the case of a CP-invariant stochastic process [5]. In this problem, the index "0" denotes vacancies, " 1 " is the index for particles and "2" for anti-particles. This implies supplementary conditions on the rates. If we are interested in the case where the CP-symmetry is explicitly broken [37, 38] one can use the solutions corresponding to the cases 2), 3) and 5) described above. In all three cases the $D_{\alpha}$ 's have the form

$$
D_{\alpha}=x_{\alpha} E_{\alpha}\left(x_{\alpha} \neq 0\right), \quad D_{\alpha}=E_{\alpha}\left(x_{\alpha}=0\right)
$$

where $E_{\alpha}$, which can be extracted from the formulae given above and are given explicitly in ref. [24], depend only on the bulk rates. The boundary rates enter in the expressions of $x_{\alpha}$ and through the fact that they have to "match" the bulk rates (see eqs. (7.48) and (7.52)). This observation allows us to see the important parameters of the problem.

Before closing this section, let us note that the case where only the cofactors $\mathcal{L}_{0}$ and $\mathcal{R}_{0}$ vanish can be treated in a similar way. In this case, the algebras satisfied by the $\mathcal{A}$ and $\mathcal{B}$ matrices (see equations (7.9a) and (7.28)) contain $\mathcal{A}^{2}$ and $\mathcal{B}^{2}$ terms as well. Fock representations for this case are also known [9].

\section{Lower rank boundary intensity matrices}

In sections 6 and 7 the Fock representations were defined by four conditions (see eqs. (5.24), (6.4) or (7.2)). The number of conditions depends on the rank of the boundary intensity matrices. In principle, one should examine all the possibilities enumerated in eqs. (4.6-12) for both left and right intensity matrices. This is a long exercise. The main point is that once 
either $L_{\mu}^{\nu}$ or $R_{\mu}^{\nu}$ or both have all the principal minors to be zero, the number of conditions is smaller and one has more freedom for the bulk rates. We shall give only four examples.

(a). $\mathcal{L}_{1} \neq 0$, all other cofactors zero.

We take the following to be the boundary intensity matrices:

$$
L=\left(\begin{array}{ccc}
-L_{1}^{0}-L_{2}^{0} & 0 & L_{0}^{2} \\
L_{1}^{0} & 0 & L_{1}^{2} \\
L_{2}^{0} & 0 & -L_{1}^{2}-L_{0}^{2}
\end{array}\right) \quad \text { and } \quad R=\left(\begin{array}{ccc}
-R_{1}^{0} & R_{0}^{1} & 0 \\
R_{1}^{0} & -R_{0}^{1} & 0 \\
0 & 0 & 0
\end{array}\right)
$$

We set $\xi=1$ in eqs. (5.8) and (5.9) to get

$$
\begin{aligned}
x_{0} & =-R_{0}^{1} \mathcal{L}_{1}, \\
x_{1} & =R_{0}^{1} \mathcal{L}_{1}, \\
x_{2} & =0 \\
\delta_{0} & =R_{0}^{1}\left(L_{1}^{2}+L_{0}^{2}\right), \\
\delta_{1} & =R_{1}^{0}\left(L_{1}^{2}+L_{0}^{2}\right)+\mathcal{L}_{1}, \\
\delta_{2} & =R_{0}^{1} L_{2}^{0} .
\end{aligned}
$$

We get several constraints on the various parameters $v_{i j}$ and $u_{k}$ that appear in the algebra (5.22) and are given in terms of the bulk and boundary rates in (5.19-20). From the condition $x_{2}=0$, and by sandwiching (5.22) between $\langle 0|$ and $|0\rangle$, we obtain

$$
v_{21}=u_{0} ; \quad v_{20}=u_{1}=0 .
$$

From $\left\langle 0\left|H_{1} H_{2} H_{0} H_{2}\right| 0\right\rangle$ we obtain

$$
v_{10}=0
$$

from which, on calculating $\left\langle 0\left|H_{1} H_{0} H_{2}\right| 0\right\rangle$, we get $q_{1}=q_{0}=q$. The correlator $\left\langle 0\left|H_{1} H_{0} H_{1} H_{2}\right| 0\right\rangle$ gives

$$
q_{2}=1+\frac{v_{01}}{u_{0}}(q-1)
$$

so we end up with

$$
\begin{array}{llll}
\frac{x_{0}}{\delta_{0}} & =\left(g_{01}-g_{10}\right) & = & \left(g_{02}-g_{20}\right)=\left(g_{21}-g_{12}\right) \\
v_{12} & =v_{02} & = & (1-q)
\end{array}
$$

The algebra is of a similar form as case c) of section 7 (eq. 7.26), apart from a relabelling of the indices and the presence of an additional constant 
term:

$$
\begin{array}{ccc}
q H_{1} H_{2}-H_{2} H_{1} & = & v_{21} H_{2}+(1-q) H_{1}+v_{21} \\
q H_{2} H_{0}-H_{0} H_{2} & = & (1-q) H_{0} \\
q_{2} H_{0} H_{1}-H_{1} H_{0}= & v_{01} H_{0}+u_{2}
\end{array}
$$

Introducing

$$
H_{0}=\frac{u_{2}}{v_{01}} \mathcal{A}, \quad H_{1}=v_{21} \mathcal{B}, \quad H_{2}=(q-1) \mathcal{N}, \quad \lambda=q_{2}^{-1} \text { and } \gamma=-\frac{g_{02}}{g_{01}}
$$

we get (compare with eq. (7.28)):

$$
\begin{array}{rlc}
\mathcal{A B}-\lambda \mathcal{B A} & = & \gamma(1+\mathcal{A}) \\
\mathcal{A N}-q \mathcal{N} \mathcal{A}= & \mathcal{A} \\
\mathcal{N B}-q \mathcal{B N}= & \mathcal{B}-\mathcal{N}+\frac{1}{1-q}
\end{array}
$$

Also, instead of four Fock conditions as in section 7, we have only three:

$$
\langle 0|\mathcal{A}=\langle 0|\mathcal{N}=0, \quad \mathcal{B}| 0\rangle=\alpha \mathcal{A}| 0\rangle
$$

with

$$
\alpha=\left(\frac{g_{02} \delta_{0}}{x_{0}\left(\delta_{1}-\delta_{0}\right)}\right)\left(\frac{\delta_{0}}{\delta_{1}} \frac{R_{1}^{0}}{R_{0}^{1}}\right) .
$$

We have not looked for explicit represenations of this algebra.

(b) All the cofactors are zero.

We consider the case when only the following four boundary rates do not vanish:

$$
L_{2}^{0}, L_{0}^{2}, R_{0}^{1} \text { and } R_{1}^{0}
$$

We find from (5.9)

$$
x_{\mu}=0 \quad(\mu=0,1,2)
$$

and the algebra is a known quadratic algebra (see section 3)

$$
q_{0} D_{1} D_{2}=D_{2} D_{1}, \quad q_{1} D_{2} D_{0}=D_{0} D_{2}, \quad q_{2} D_{0} D_{1}=D_{1} D_{0},
$$

with only two conditions:

$$
D_{1}|0\rangle=\frac{R_{1}^{0}}{R_{0}^{1}} D_{0}|0\rangle \text { and }\langle 0| D_{2}=\frac{L_{2}^{0}}{L_{0}^{2}}\langle 0| D_{0} .
$$

All vacuum expectation values of degree $L$ can be expressed in terms of $\left\langle 0\left|D_{0}^{L}\right| 0\right\rangle$. The appearance of the polynomial algebras in this new context is 
very interesting since, as shown in section 3, we know plenty of them, and not just for the simple exclusion processes. This opens up the possibility of a new class of solutions for more general processes with open ends.

(c) All the cofactors are zero, but the non-zero boundary rates are:

$$
L_{1}^{0}, L_{2}^{0}, R_{0}^{1} \text { and } R_{1}^{0} \text {. }
$$

In this case the algebra is also given by $(8.11)$ and $x_{\mu}=0,(\mu=0,1,2)$, but the generators satisfy the conditions

$$
\langle 0| D_{0}=0 \text { and } D_{1}|0\rangle=\frac{R_{1}^{0}}{R_{0}^{1}} D_{0}|0\rangle .
$$

It is interesting to observe that no boundary condition appears for the operator $D_{2}$. Using (8.11) and (8.14) it is simple to see that the ground state will have a the single word $D_{2}^{L}$, which corresponds to the lattice filled by particle "2". This can be expected physically from (8.13) since the species " 2 " is never removed from the chain at either end, unlike the species " 0 " and " 1 ", and is created at the left end with a certain rate $L_{2}^{0} \neq 0$. Therefore, when the time goes to infinity the system will end up with only particles of species " 2 ".

(d) All cofactors are zero, but the non-zero boundary rates are:

$$
L_{1}^{0}, L_{2}^{0}, R_{1}^{0} \text { and } R_{2}^{0} \text {. }
$$

The algebra is again given by (8.11) but the conditions are

$$
\left\langle 0\left|D_{0}=D_{0}\right| 0\right\rangle=0,
$$

and no conditions for $D_{1}$ and $D_{2}$. In this case we have $L$ degenerate ground states expressed in terms of the words $D_{1}^{n} D_{2}^{L-n}(n=1,2, \ldots, L)$. This is expected physically from (8.15) since in the large time limit, we should expect no " 0 " particles and the particles " 1 " and " 2 " are conserved separately.

\section{Conclusions}

This was a long journey. We will first list the mathematical results which are independent of the physical applications. In section 3 we have given a list of polynomial algebras defined by a set of homogenous quadratic relations (see for example eqs.(3.25-27)). In sections 6 and 7 we have given Fock 
representations for the algebra defined by eq.(5.22). Those are a special class of the inhomogenous quadratic algebras defined by eq.(1.24). The representations are one dimensional (see eq.(5.16)), two-dimensional for the "generic" case described in section 6, or infinite dimensional (see the five special cases of the algebra (7.36)). These algebras can be useful for physical applications other than for the case of open chains considered in this paper. For example we used them ourselves while studying stochastic processes on a ring [24]. As for the physical applications relevant to this paper, we have looked for steady states of stochastic processes defined by bulk and boundary intensity matrices (see eq. (1.1)). The probability distribution of the steady states is given by the expression (1.13) for chains with open ends.

In our approach the same expression without $|0\rangle$ is used for a left open end and a closed right end. In the case of closed chains we have to drop the $\langle 0|$ and $|0\rangle$ symbols. Polynomial algebras are defined by taking the $X$ 's equal to zero in eq.(1.11). They exist only for special choices of the bulk rates. A given polynomial algebra can be used in three places: for closed ends (see section 3), for the left end open and the right end closed when supplementary restrictions come from the first equation in (1.12) with the $X$ 's are taken to zero (see section 4) and even for the case of a chain with open ends (see the example in section 8, eqs.(8.11-12)). As far as we know it is the first time that the problem with one or two closed ends is solved using algebraic methods.

Most of this paper is dedicated to the problem of the chain with open ends where we have taken c-numbers for the $X$ 's in eqs.(1.11) and (1.12). We have confined ourselves to the simpler problem of exclusion processes. In solving this problem a crucial point is the rank of the boundary intensity matrices since they dictate the number of conditions which define the Fock representations. In the 3 state problem, they can be four (see sections 6 and 7) three or two (see section 8). In the case of four conditions an important role is played by the number of cofactors of the boundary intensity matrices which vanish. All in all we have given many examples which can be used for physical applications. It is obvious how the present method may be generalized for four or more states problems. The case of periodic boundary conditions is considered in 24].

After writing this paper, one can ask where lies the mathematical beauty. Probably in the simpler cases where polynomial algebras can be used and in the algebras described in section 7 . The problem of open boundaries with two states depends on 5 parameters; the one with three states on 17 parameters and there is a price to pay in order to find solutions. In this 
paper we have never properly exploited the Krebs-Sandow theorem (eqs. (1.12-15)) since we have always chosen representations of the $X_{\alpha}$ 's to be scalars or zero. The reason is that we wanted to have quadratic algebras that are understood, like the polynomial algebras or those of type (1.24), and we did not find new ones.

We would like to make one last comment which has to do with the connection between finding the ground states by algebraic methods and the integrability of the Hamiltonian. As is shown in section 3 several exactly integrable Hamiltonians, connected with stochastic dynamics, can have their ground state wave function written in terms of words defined by algebraic relations. But in general, the existence of an algebraic form of the ground state wave function is not a sufficient condition in order to ensure exact integrability.

\section{Acknowledgements}

The work of F.C.A. was supported in part by FAPESP and CNPQ Brazil. The work of S.D. was supported in part by the EPSRC grant GRJ25758, and he would like to thank Universität Bonn for their hospitality. V.R. would like to thank the hospitality of the Weizmann Institute, City University, the Federal University of São Carlos, the Institute for Theoretical Physics at the UCSB, and especially of SISSA where this work was done, and also the DAAD, the FAPESP, the National Science Foundation under the Grant No. PHY94-07194 and the EC TMR Programme for finan-

cial support. We would like to thank P.F. Arndt, L. Dabrowski, B. Derrida, T. Heinzel, K. Krebs, D.Mukamel, V. Pasquier, M. Scheunert and especially P.P. Martin for discussions.

\section{References}

[1] J. Krug and H. Spohn, Solids far from Equilibrium, ed. C.Godreche (Cambridge, Cambridge University Press 1991).

[2] B. Derrida, E. Domany and D. Mukamel, J. Stat. Phys. 69 (1992) 667. 
[3] E. Domany and G. Schütz, J. Stat. Phys. 72 (1993) 277.

[4] B. Derrida, M. R. Evans, V. Hakim and V. Pasquier, J. Phys. A 26 (1993) 1493.

[5] M. R. Evans, D. P. Foster, C. Godrèche and D. Mukamel, J. Stat. Phys. 80 (1995) 69.

[6] B. Derrida, J. L. Lebowitz and E. R. Speer, J. Stat. Phys. (1997) to be published.

[7] K. Nagel and M. Schreckenberg, J. Physique 2 (1992) 2221.

[8] D. L. Isaacson and P. W. Madson, Markov Chains: Theory and Applications, Wiley Series in Probability and Mathematical Statistics, 1976. Marius Iosifescu, Finite Markov processes and applications, (Wiley, Chichester, 1981).

[9] F. H. L. Essler and V. Rittenberg, J. Phys. A 29 (1996) 3375.

[10] F. C. Alcaraz and V. Rittenberg, Phys. Lett. B 314 (1993) 377.

[11] K. Krebs and S. Sandow, J. Phys. A (1997), to be published.

[12] M. B. Zvyagina, J. Sov. Math. 41 (1988) 992.

[13] A. M. Vershik, Selecta Mathematica Sovietica 11 (1992) 293.

[14] H. Hinrichsen, I. Peschel and S. Sandow, J. Phys. A 29 (1996) 2643.

[15] K. Krebs, S. Sandow and H. Simon, to be published.

[16] H. Hinrichsen, J. Phys. A 29 (1996) 3659.

[17] A. Honecker and I. Peschel, cond-mat/9606053.

[18] N. Rajewsky, A. Schadschneider and M. Schreckenberg, cond-mat/9603172.

[19] V. Hakim and J. P. Nadal, J. Phys. A 16 (1983) L 213.

[20] M. Fannes, B. Nachtergale and R. F. Werner, Rev. Bras. de Fisica 19 (1989) 460; Comm. Math. Phys. 174 (1996) 477, and references therein.

[21] H. Niggemann, A. Klümper and J. Zittartz, cond-mat/9702178 and references therein. 
[22] B. Derrida, S. A. Janowski, J. L. Lebowitz and E. R. Speer, J. Stat. Phys. 73 (1993) 813.

[23] K. Mallick, J.Phys. A 29 (1996) 5375.

[24] P. F. Arndt, T. Heinzel and V. Rittenberg, cond-mat/9703182.

[25] Yu. I. Manin, Comm. Math. Phys. 123 (1989) 163.

[26] A. Sudbery, J. Phys. A 23 (1990) L 697.

[27] N. Yu.Reshetikhin, L. A. Takhtadzyan and L. D. Faddeev, Leningrad Math. J. 11 (1990) 193.

[28] V. F. R. Jones, Int. J. Mod. Phys. B 4 (1990) 701.

[29] R. J. Baxter, Exactly solved models in statistical mechanics (Academic Press, New York 1982)

[30] P. P. Martin, Potts models and related problems in statistical mechanics (World Scientific, Singapore 1991)

[31] J. H. H. Perk and C. L. Schultz, Phys. Lett. A 84 (1981) 407.

[32] P. P. Kulish and E. K. Sklyanin, Lecture Notes in Physics, vol. 151 (Springer, Berlin 1982) p. 61.

[33] F. C. Alcaraz, Int. J. Mod. Phys. B 8 (1994) 3449.

[34] P. P. Martin and V. Rittenberg, Int.J.Mod.Phys. A7, Suppl.1 B (1992) 797 and references therein.

[35] F. C. Alcaraz, D. Arnaudon, V. Rittenberg and M. Scheunert Int. J. Mod. Phys. A9 (1994) 3473 and references therein.

[36] G. Schütz and S. Sandow, Europhysics Letters 26 (1994) 7.

[37] P. F. Arndt, T. Heinzel and V. Rittenberg, SISSA 39/97/EP.

[38] P. F. Arndt and T. Heinzel, in preparation. 


\section{A The CP-invariant steady state}

It was recently shown that in steady states $\mathrm{CP}$-invariance can be spontaneously broken [5] and that the phase diagram cannot be obtained by mean field methods [24]. This is not the right place to describe the physics of the problem and the interested reader should have a look at the references. The model where this invariance is spontaneously broken is a special case of the problem studied in section 7 , where the rates we choose are $\mathrm{CP}$ invariant. Charge conjugation (C) is defined by the operation $1 \rightarrow 2,2 \rightarrow 1$ and $0 \rightarrow 0$, while the parity $(\mathrm{P})$ operator corresponds to interchanging left and right.

The non-vanishing boundary rates are

$$
\begin{gathered}
L_{1}^{2}=R_{2}^{1}, \quad L_{1}^{0}=R_{2}^{0}, \quad L_{2}^{0}=R_{1}^{0}, \quad L_{0}^{2}=R_{0}^{1} \\
\mathcal{L}_{1}=\mathcal{R}_{2}, \quad \mathcal{L}_{0}=\mathcal{L}_{2}=\mathcal{R}_{0}=\mathcal{R}_{1}=0 .
\end{gathered}
$$

The bulk rates are

$$
g_{10}=g_{02}, \quad g_{01}=g_{20}, \quad q_{1}=q_{2}=q,
$$

and $g_{12}$ and $g_{21}$ are arbitrary.

In ref. [5] a special choice was taken:

$$
L_{1}^{0}=R_{2}^{0}=\alpha \quad \text { and } \quad L_{0}^{2}=R_{0}^{1}=\beta,
$$

with all other boundary rates set to zero. The non-vanishing bulk rates were taken to be $g_{12}=g_{10}=g_{02}$. Here we consider the general case.

From equations (7.43), (7.44) and (5.17) and choosing $\xi^{-1}=\left(L_{0}^{2}+2 L_{1}^{2}\right)$ we obtain

$$
\begin{aligned}
& \delta_{0}=L_{0}^{2}, \quad \delta_{1}=\delta_{2}=\left(L_{1}^{0}+L_{2}^{0}\right) \\
& y_{0}=0, \quad y_{1}=-y_{2}=\frac{L_{1}^{0} L_{0}^{2}}{L_{1}^{0}+L_{2}^{0}}+L_{1}^{2} .
\end{aligned}
$$

We now use the results of section 7 (equations (7.43)-(7.55)). From eq. (7.45) and (A.4) we have

$$
\frac{L_{1}^{0} L_{0}^{2}}{L_{1}^{0}+L_{2}^{0}}+L_{1}^{2}=g_{10}-g_{01}
$$

We now consider the cases (2), (3) and (5) separately.

Case (2), $g_{20}=g_{01}=0$.

We use equations (7.47) and (7.48) to get

$$
\frac{L_{1}^{0} L_{0}^{2}}{L_{1}^{0}+L_{2}^{0}}+L_{1}^{2}=g_{10}
$$


and

$$
\begin{array}{cc}
v_{10}=v_{20}=1, & v_{01}=v_{02}=0, \\
v_{12}=v_{21}=\frac{g_{10}+g_{21}-g_{12}}{g_{21}}, & u_{0}=\frac{2 g_{10}+g_{21}-g_{12}}{g_{21}} .
\end{array}
$$

Using eq. (5.21) it is easy to show that

$$
D_{1}=\left(\frac{L_{1}^{0}+L_{2}^{0}}{L_{0}^{2}}\right)\left(1+H_{1}\right), \quad D_{2}=\left(\frac{L_{1}^{0}+L_{2}^{0}}{L_{0}^{2}}\right)\left(1+H_{2}\right) .
$$

Introducing

$$
\lambda=\frac{1}{q_{0}}=\frac{g_{21}}{g_{12}} \quad \text { and } \quad \omega=\frac{g_{10}}{g_{12}}
$$

we have

$$
H_{1}=H_{2}^{T}=\sqrt{2 \omega+\lambda-1}\left(\begin{array}{cccc}
u_{1} & v_{1} & 0 & \ldots \\
0 & u_{2} & v_{2} & \ldots \\
0 & 0 & u_{3} & \ldots \\
\vdots & \vdots & \vdots & \ddots
\end{array}\right), \quad D_{0}=\left(\begin{array}{cccc}
1 & 0 & 0 & \ldots \\
0 & 0 & 0 & \ldots \\
0 & 0 & 0 & \ldots \\
\vdots & \vdots & \vdots & \ddots
\end{array}\right)
$$

where

$$
\begin{aligned}
& v_{n}^{2}=\{n\}_{\lambda}\left(1+\frac{(\omega+\lambda-1)^{2}}{(2 \omega-1+\lambda)}\{n-1\}_{\lambda}\right) \\
& u_{n}=\frac{(\omega+\lambda-1)}{\sqrt{2 \omega-1+\lambda}}\{n-1\}_{\lambda} .
\end{aligned}
$$

Notice that the boundary conditions appear only in (A.6) and in the normalization factors of $D_{1}$ and $D_{2}$. They do not change the physics of the problem which is governed by $\omega$ and $\lambda$. In other words, taking only the rates (A.3) or the general case (A.1) does not change the physical results.

In ref. [5] only the case $\lambda=0$ was considered.

Case $(3) g_{10}=g_{02}, g_{01}=g_{20}$ and $g_{12}-g_{21}=g_{10}-g_{01}$.

We make use of eqs. $(7.49-52)$. The bulk rates and the boundary rates are related by the constraint:

$$
g_{10}-g_{01}=\frac{L_{1}^{0} L_{0}^{2}}{L_{1}^{0}+L_{2}^{0}}+L_{1}^{2}
$$


We then have

$$
\begin{array}{rlrl}
D_{1} & =\frac{L_{1}^{0}+L_{2}^{0}}{L_{0}^{2}}(1+R) & D_{2}=D_{1}^{T} \\
R & =\left(\begin{array}{cccc}
0 & r_{1} & 0 & \ldots \\
0 & 0 & r_{2} & \ldots \\
0 & 0 & 0 & \ldots \\
\vdots & \vdots & \vdots & \ddots
\end{array}\right), D_{0}=\left(\begin{array}{cccc}
s_{1} & 0 & 0 & \ldots \\
0 & s_{2} & 0 & \ldots \\
0 & 0 & s_{3} & \ldots \\
\vdots & \vdots & \vdots & \ddots
\end{array}\right),
\end{array}
$$

where

$$
r_{k}^{2}=1-\lambda^{k} \quad \text { and } \quad s_{k}=1-\{k-1\}_{q}
$$

with

$$
\lambda=\frac{g_{21}}{g_{12}} \quad \text { and } \quad q=\frac{g_{20}}{g_{02}}=\frac{g_{01}}{g_{10}} .
$$

Notice once more that the boundary terms are not essential and in this case as well, the conditions (A.3) capture the whole physics.

Case (5) (see eqs. (7.53-55)).

Since $g_{02}=g_{10}$, we get $v_{12}=0$ (see eq. (7.55)) which together with (7.41) brings us back to case (3).

In conclusion, considering the most general boundary rates (four instead of two) does not change the domain of applications of the algebraic approach. What we have shown however, is that it can be applied for a larger choice of bulk rates [38]. 